\title{
Patterns in CAOSS: Distributed representations predict variation in relational interpretations for familiar and novel compound words
}

\author{
Fritz Günther ${ }^{1} \&$ Marco Marelli ${ }^{2,3}$ \\ ${ }^{1}$ Humboldt-Universität zu Berlin, Berlin, Germany \\ ${ }^{2}$ University of Milano-Bicocca, Milan, Italy \\ ${ }^{3}$ NeuroMI, Milan Center for Neuroscience, Milan, Italy
}

\begin{abstract}
Note: This is the author's preprint version of the article (date: 28. 02. 2022). The final article is accepted for publication in Cognitive Psychology.
\end{abstract}

\begin{abstract}
While distributional semantic models that represent word meanings as high-dimensional vectors induced from large text corpora have been shown to successfully predict human behavior across a wide range of tasks, they have also received criticism from different directions. These include concerns over their interpretability (how can numbers specifying abstract, latent dimensions represent meaning?) and their ability to capture variation in meaning (how can a single vector representation capture multiple different interpretations for the same expression?). Here, we demonstrate that semantic vectors can indeed rise up to these challenges, by training a mapping system (a simple linear regression) that predicts inter-individual variation in relational interpretations for compounds such as wood brush (for example brush FOR wood, or brush MADE OF wood) from (compositional) semantic vectors representing the meanings of these compounds. These predictions consistently beat different random baselines, both for familiar compounds (moon light, Experiment 1) as well as novel compounds (wood brush, Experiment 2), demonstrating that distributional semantic vectors encode variations in qualitative interpretations that can be decoded using techniques as simple as linear regression.
\end{abstract}

Keywords: Distributional semantics; Compound words; Conceptual combination; Relational information; Novel compounds

Since the inception of prominent exemplars such as Latent Semantic Analysis (LSA; Landauer \& Dumais, 1997), distributional semantic models have become increasingly popular as computational models of word meaning (Jones, Willits, \& Dennis, 2015). The core idea of these models is to take large corpora of natural language as proxies for the language experience of a speaker (population), from which learning algorithms can induce semantic representations. Based on the distributional hypothesis that words with similar meanings tend to occur in similar contexts (Harris, 1954; Lenci, 2008), numerical vectors encoding the distributions of words over pre-defined contexts (for example, their

This study was supported was supported by Grant 2017-ATE0291 from the Department of Psychology, University of Milano-Bicocca, awarded to Marco Marelli, and received ethical approval by the Departmental Commission for Research Evaluation of the Department of Psychology (CRIP), University of MilanoBicocca (project RM-2019-223). Data and analysis scripts are available at https://osf.io/ycd64/. The authors declare that no competing interests exist. surrounding words or the documents they appear in) are then taken as quantitative representations of word meaning. Distributional models have several appealing properties - most notably, a high performance across a range of linguistic and cognitive tasks (Baroni, Dinu, \& Kruszewski, 2014; Pereira, Gershman, Ritter, \& Botvinick, 2016) - and have been forwarded as promising candidates for fully-implemented, datadriven computational models of human semantic memory (Jones et al., 2015; Mandera, Keuleers, \& Brysbaert, 2017). Most importantly, on a theoretical level, these approaches allow us to rigorously model and investigate the role of language experience in shaping our conceptual system and the mental representations of objects in the world (Günther, Rinaldi, \& Marelli, 2019).

Recent developments have further raised the stake of distributional semantic models, as models implementing compositional operations have become more and more popular (Baroni, Bernardi, \& Zamparelli, 2014; Marelli, Gagné, \& Spalding, 2017; Mitchell \& Lapata, 2010). Such systems are able to induce semantic vectors for unattested word combinations on the basis of corpus data (often by adding up (transformations of) the constituent vectors; Marelli et 
al., 2017; Mitchell \& Lapata, 2010), opening the distributional approach to properly capture the remarkable expressive power of natural language. Indeed, compositional distributional models were shown to align with human intuitions about word combinations (Günther \& Marelli, 2016; Vecchi, Marelli, Zamparelli, \& Baroni, 2017), and they were able to replicate a number of well-known effects in the psychological literature (see Marelli et al., 2017).

However, at first glance the meaning representations induced by distributional models can appear quite unsatisfactory, especially from a psychological point of view: Each word (no matter if simplex or complex) is distilled into a single list of numbers (i.e., a high-dimensional vector). And, as stated by Kintsch (2001), one of the early proponents of distributional models in cognitive science: "All we can do, however, is compare one vector with another. Inspecting the 300 numbers that compose it tells us little, for the dimensions of the semantic space are not identifiable. The only way we can tell what a given vector means is to find out what other words or sentence vectors are close to it." (p. 260). This is markedly different from other approaches such as featurebased models (e.g. McRae, Cree, Seidenberg, \& McNorgan, 2005), whose representations have inherently interpretable dimensions. In addition, distributional models typically only consider word forms or lemmata (for a discussion, see Turney \& Pantel, 2010), thus packing all possible meanings associated to a given label (for example ball or animal doctor) into a single vector and, in turn, a single point in semantic space. In other words, these models seem to conflate different meanings and senses into a single averaged representation, risking to lose (especially less frequent) subordinate senses (Jamieson, Avery, Johns, \& Jones, 2018).

In the present paper, we argue that these central issues of interpretability and variation in meaning can be addressed within the distributional semantics framework, by demonstrating that different qualitative semantic interpretations by different speakers can be decoded from (and are thus encoded in) distributional vectors. In the next section, we will describe these two central issues in more detail, while illustrating how they pertain to compound words (as a special case of compositional expressions) in particular, which thus serve as an ideal test case for our study.

\section{Challenges to distributional semantic models - inter- pretability and variation in meaning}

While they have enjoyed empirical as well as theoretical support (as outlined above), distributional models have also received criticism from many different directions (for a recent overview, see Günther et al., 2019). One main point of contention is the issue described in the quote by Kintsch (2001) above, and typically expressed in the argument that these models represent word meanings as a list of numbers indicating the values of abstract, un-interpretable dimensions (e.g., Borghesani \& Piazza, 2017), and thus "devoid of content" (Rogers \& Wolmetz, 2016, p. 124). This assessment is further reinforced by the fact that the most prominent application for these models is to compute similarities between words, leading some to the impression that they can only define word meanings through their connection to other word meanings (Glenberg \& Robertson, 2000; Kintsch, 2001).

In a prominent, recent position paper, Lake and Murphy (2021) use exactly this line of argument to challenge the psychological validity of distributional semantic models by questioning their ability to adequately capture conceptual combination (see Murphy, 2002; Ran \& Duimering, 2009, for reviews of theories on conceptual combination), one of the main phenomena that any comprehensive theory of semantic representation needs to be able to explain (Murphy, 2002). These authors argue that, as all distributional semantic vectors, vectors for complex expressions like "apartment dog" that are created out of the vectors for their two constituents are inherently inscrutable, and that "there is no easy way to find out how the model is interpreting "apartment dog", even if we know that its interpretation is similar to that of "tame lion", say." (Lake \& Murphy, 2021, p. 19). These authors thus perpetuate the argument by Kintsch (2001) that the only possibility to understand distributional vectors is to compare them to one another (adding the that this is to be considered insufficient). The arguments against the adequacy of compositional distributional models is then further specified with respect to the relation between the compound constituents" : "For example, an ocean view is a view where one can see the ocean, whereas an ocean wave is a wave of the ocean. It's difficult to see how adding the same exact ocean vector to each head noun could produce such a wide range of semantic transformations (Lake \& Murphy, 2021, p. 20)."

As highlighted by this argument, compound words referring to combined concepts such as "ocean view" or "apartment dog" are indeed a highly interesting test case for qualitative interpretations of semantic representations, because the meaning of compounds can be characterized via a very specific type of qualitative information: the semantic relation linking together the two constituents (Gagné, 2000; Levi, 1978). Of course, these relations do not fully describe the meaning of a compound, but they define important meaning aspects by imposing restrictions on the number of possibilities for more fine-grained interpretations. For example, an "apartment dog" can be classified as a "dog LOCATED IN an apartment", or a "dog FOR an apartment", but not really as a "dog MADE OF an apartment" in the way that a "plastic dog" could be a "dog MADE OF plastic". In linguistic theories, these relations are usually con-

\footnotetext{
${ }^{1}$ In the English language, the head is almost always the second constituent in a compound that defines its semantic category, and the modifier is the first constituent; Williams, 1981.
} 
sidered as part of a fairly well-defined, closed set of possible relations, although there is substantial debate around these taxonomies (Allen, 1978; Gagné, 2000; Levi, 1978; Jackendoff, 2009; Schmidtke, Gagné, Kuperman, Spalding, \& Tucker, 2018). Over and above their importance for the linguistic classification of compounds, a long line of research has also demonstrated that these relations play an important role in the psychological representation and processing of conceptual combinations (Gagné \& Shoben, 1997; Gagné, 2000; Gagné, Spalding, Figueredo, \& Mullaly, 2009; Gagné \& Spalding, 2009; Schmidtke, Gagné, Kuperman, Spalding, \& Tucker, 2018; Schmidtke, Kuperman, Gagné, \& Spalding, 2016; Spalding, Gagné, Mullaly, \& Ji, 2010). Most prominently, the CARIN model (Gagné \& Shoben, 1997) and its successor, the RICE model (Spalding et al., 2010), posit that the processing and interpretation of (familiar and novel) compounds involves a competition between different possible relations linking the two compound constituents. More specifically, these models assume that multiple competing relational interpretations for the compound are constructed and then evaluated for plausibility. The possible relations are suggested by the modifier semantics (for example, "chocolate" is most commonly associated with a MADE OF relation, and more rarely with a FOR or ABOUT relation), and the semantic information provided by the head is then used to evaluate the plausibilities of these possible relations (see Gagné \& Spalding, 2013, for a review). However, sharing the doubts expressed in the quotes by Lake and Murphy (2021) cited earlier, Lynott and Ramscar (2001, p. 9) already suspected that a distributional semantic model "cannot provide a complete account of conceptual combination, since it provides no explicit information on the relationships between heads and modifiers".

This already challenging state of affairs is further complicated by the psychological reality of conceptual combination, which is more complex and nuanced than the linguistic ideal of assigning a unique relation to each compound (Bell, 2015; Schäfer \& Bell, 2020; Schmidtke, Gagné, Kuperman, Spalding, \& Tucker, 2018; Schmidtke et al., 2016). This is already illustrated by the "apartment dog" example in the previous paragraph, which is far from being an exception: While most speakers agree that "teatime" is interpreted as "time FOR tea", there is significant disagreement, for example, for "folksong", for which the interpretations "song ABOUT folk", "song BY folk", "song FOR folk" are given by the same proportion of speakers, alongside some other less-frequent interpretations such as "song USED BY folk" (Spalding \& Gagné, 2014; Schmidtke, Gagné, Kuperman, Spalding, \& Tucker, 2018; Schmidtke et al., 2016). In several large-scale empirical studies, Schmidtke and colleagues have demonstrated that this variability in relational interpretation (characterized in their studies as relational entropy, a measure describing how close the distribution of these in- terpretations is to a uniform distribution) even affects processing times for compound words (see also Spalding \& Gagné, 2014), with processing costs for high-entropy words such as folksong (Schmidtke, Gagné, Kuperman, Spalding, $\&$ Tucker, 2018; Schmidtke et al., 2016). These results have been interpreted in line with the CARIN (Gagné \& Shoben, 1997) and RICE (Spalding et al., 2010) models of conceptual combination. The studies by Schmidtke and colleagues only employed familiar compounds; however, if there already is disagreement for familiar words, the phenomenon should only be more pronounced for novel conceptual combinations such as "apartment dog" or "ice dog" (Ryder, 1994; Schäfer \& Bell, 2020).

This variation in interpretation is in fact another central empirical phenomenon that has been advanced as a main point of criticism against distributional semantic models: If these models represent each word meaning as a single numerical vector, how can they at the same time represent different possible readings of the same word or phrase (see Camacho-Collados \& Pilehvar, 2018; Heylen, Wielfaert, Speelman, \& Geeraerts, 2015; Jamieson et al., 2018, for further discussions with possible solutions)? Especially when words are used in new contexts and combinations that the model has never encountered before (French \& Labiouse, 2002; Glenberg \& Robertson, 2000)?

As we described above, interpretational variability is a highly prevalent phenomenon especially for compound words (as compared to simplex words): compound interpretations are inherently ambiguous (Gagné \& Shoben, 2002; Spalding \& Gagné, 2014), even when their constituents are completely monosemous. Combining two ambiguous words tends to only further amplify this inherent interpretational ambiguity, with constituent polysemy strongly contributing to higher interpretational variability in compound words (Schäfer \& Bell, 2020). This will be especially relevant for novel compounds, which do not even have canonical interpretations that can exert a certain influence on speakers' interpretations. Taken together, these points further demonstrate that compounds are a particularly well-suited test case for our investigations.

\section{The present study}

In the present article, we demonstrate that distributional semantic models can in fact stand up to the challenges described, by demonstrating that variations in speakers' qualitative interpretations for compounds can be predicted from (and are thus implicitly encoded in) their semantic vectors. As outlined above, compounds offer an ideal test case, because they allow us to address all points simultaneously: Variations in qualitative meaning interpretations can be directly quantified via distributions over the closed set of possible relational structures, both for familiar ("clam shell") and for completely novel combinations ("sword bird"). 
In our endeavor, we follow the general approach outlined by Bell and Schäfer (2013): On the topic of modeling compound meanings in a distributional semantics approach, these authors state that "with regard to semantic relations, the situation is a bit more challenging. A fairly straightforward first step might be to use a compound classification algorithm on the data" (p. 9). While subsequent studies in the field of computational linguistics have developed such classifiers (Dima \& Hinrichs, 2015; Shwartz \& Dagan, 2018; Verhoeven \& Daelemans, 2013), these studies aim at predicting the normative linguistic ideal of a single "correct" relation per compound, thus missing the psychological reality of substantial variation in compound interpretation (Schmidtke et al., 2016; Schmidtke, Gagné, Kuperman, Spalding, \& Tucker, 2018; Spalding \& Gagné, 2014). In the first experiment, we thus extend upon this work by demonstrating that even the relational distributions of compounds (Schmidtke, Gagné, Kuperman, Spalding, \& Tucker, 2018) can indeed be predicted from the dimensional values of their respective semantic vectors. We achieve this by training a simple linear-regression mapping system between semantic vectors and relational distributions. In the second experiment, we then show that this generalizes to novel conceptual combinations with which neither human speakers nor the distributional model have prior experience.

\section{Experiment 1: Familiar compounds}

Data and analysis scripts for Experiment 1 as well as Experiment 2 are available at https://osf.io/ycd64/.

\section{Dataset}

The dataset for our first study was collected by Schmidtke, Gagné, Kuperman, Spalding, and Tucker (2018) and consists of participant ratings on the semantic relations for 604 different familiar compound words (with 350 different modifiers and 310 different heads) that are typically used in their closed form (such as thunderstorm). In each trial of the study, participants were presented with one of the compounds, but in their open form (i.e., thunder storm instead of thunderstorm). Participants were instructed to pretend that they were learners of English who have never seen the specific combination of words before, but were familiar with both constituents as individual words. Note that both the open-form presentation and these specific instructions encouraged the participants to compositionally derive a meaning for the combined concept, instead of just purely relying on the compound lexicalized meaning.

Participants were then asked to perform a possible relations task (Spalding \& Gagné, 2014), that is, to indicate what these expressions mean by selecting one of 16 possible interpretational relations, adapted from Levi (1978) (see Table 1). For each compound, the dataset contains ratings from between 35 and 49 participants. The dataset by Schmidtke,
Gagné, Kuperman, Spalding, and Tucker (2018) is the largest dataset to date which contains such distributions of relational interpretations.

In the original study, Schmidtke, Gagné, Kuperman, Spalding, and Tucker (2018) used the distribution of ratings to compute a single measure of relational entropy (i.e., the degree to which participants agree on a single interpretation). As described in the introduction, this measure predicts processing times for the compound words, both in visual compound processing (when the compounds were presented in their closed form;) and auditory compound processing (Schmidtke, Gagné, Kuperman, Spalding, \& Tucker, 2018). Thus, the psychological validity of this dataset is thoroughly evaluated across different large-scale behavioral studies. In the present study however, the distribution itself more specifically, the frequencies of each of the 16 different relations for each compound - will serve as the dependent behavioral variable to be predicted by our mapping system.

\section{Semantic vectors}

The aim of our study was to investigate whether these explicit semantic intuitions can be predicted from the subsymbolic dimensional values of semantic vectors representing the compound meanings. These dimensional values thus serve as the independent variables of our study.

We extracted all semantic vectors from a stateof-the art model for representing lexical semantics (Lenci, Sahlgren, Jeuniaux, Gyllensten, \& Miliani, 2021), which was identified as the best-performing distributional semantic model in the systematic evaluation study by Baroni, Dinu, and Kruszewski (2014): A cbow variant of the word2vec algorithm (Mikolov, Chen, Corrado, \& Dean, 2013), aimed at predicting each target word in the corpus from the 5 words to its left and its right (i.e., window size 5), negative sampling with $k=10$, and subsampling with $t=1 e^{-5}$. The model was trained on an English $\sim 2.8$ billion word corpus (a concatenation of the ukWaC corpus, an English Wikipedia dump, and the British National Corpus).

\section{The mapping system}

We employed a least-squares multivariate multiple linear regression model to simultaneously predict the frequencies for all 16 compound relations from the 400 dimensional values of the semantic vector representing a compound meaning (see Figure 1). This is a generalization of the standard multiple regression method, where an outcome variable is predicted from a set of predictor variables; the only difference is that more than one outcome variable is predicted at the same time. This method is more efficient than the alternative of estimating one separate regression model for each outcome variable in the (very likely) case of correlations between the outcome variables (Hartung \& Knapp, 2005). Practically, it means estimating a regression weight between each predictor 
Table 1

The 16 relational interpretations, illustrated for the compound thunder storm, alongside their frequency of selection in the dataset by Schmidtke et al. (2018)

\begin{tabular}{lr|lr}
\hline relation & frequency & relation & frequency \\
\hline storm ABOUT thunder & 1 & thunder HAS storm & 1 \\
storm BY thunder & 1 & storm IS thunder & 2 \\
storm CAUSED BY thunder & 4 & storm LOCATION IS thunder & 0 \\
storm CAUSES thunder & 7 & thunder LOCATION IS storm & 1 \\
storm DURING thunder & 0 & storm MADE OF thunder & 9 \\
storm FOR thunder & 0 & storm MAKES thunder & 6 \\
storm FROM thunder & 0 & storm USED BY thunder & 0 \\
storm HAS thunder & 11 & storm USES thunder & 1 \\
\hline
\end{tabular}

and each outcome variable (the grey lines in Figure 1) and, as in standard linear regression, these are estimated by reducing the squared differences between the model predictions and the observed values.

To minimize the risk of overfitting, we employed a leaveone-out cross-validation (LOOCV) technique (see Richie, Zou, \& Bhatia, 2019; Utsumi, 2020; Zou \& Bhatia, 2021). Here, the regression model is trained on all items except for one, and then the dimensional values of this one left-out item (the test item) are passed through the regression weights in order to estimate its relational judgment frequencies. For the mapping system, the left-out item is thus treated as a compound it has never encountered before. This process is repeated for each of the 575 items in the dataset (resulting in 575 slightly different mapping systems), and the overall performance of the system will be calculated as the average performance of these 575 instances (see the Results section).

Representing compound meanings. The aim of this study is to examine to what extent the distribution of relational interpretations for a compound can be predicted from the dimensional values of a vector representing this compound's meaning. The most straightforward vector representation for each compound in the dataset is simply the vector representing its closed-form variant in this semantic model, as extracted from the corpus (i.e., all occurrences of the string snowman in the corpus are used to induce the vector representation for snow man). We refer to this model as the whole-word model. Of the 604 different compounds used by (Schmidtke, Gagné, Kuperman, Spalding, \& Tucker, 2018), vectors for 575 were included in this semantic model; our analysis will thus run on this subset of 575 compounds.

However, research on morphological processing has long established that speakers do not only represent compound meanings as lexicalized whole-word units. The defining feature of compounds is that they are built from two constituents with meanings of their own, and speakers can attempt to combine the meanings of these two constituents in order to get to a compositional meaning for the compound (Ji, Gagné, \& Spalding, 2011; Marelli \& Luzzatti, 2012). In fact, this meaning combination appears to occur automatically in compound processing (El-Bialy, Gagné, \& Spalding, 2013; Libben, 2014; Libben, Gibson, Yoon, \& Sandra, 2003; Günther \& Marelli, 2019). In most (semantically transparent) cases, this combined meaning is fairly similar to the lexicalized meaning of a compound (Gagné, Spalding, \& Schmidtke, 2019); however, in some (semantically opaque) cases, the two can diverge drastically: In its lexicalized meaning, moonshine refers to illegallyproduced liquor, but just from combining its constituents, one would obtain a compositional meaning that is very similar to moonlight.

A very simple and well-established model in the literature is the additive model (Mitchell \& Lapata, 2010), which represents compositional compound meanings $c$ as $c=u+v$ (with $u$ being the modifier vector and $v$ being the head vector). However, the additive model has some potential disadvantages - for example, since addition is a commutative operation, we would obtain the same vector representations for houseboat and boathouse, even though these two clearly have different meanings. We thus employ as an additional compositional model the CAOSS model (Compounding as Abstract Operation in Semantic Space; Marelli et al., 2017), a refined and parametrized version of the additive model that has shown good empirical performance in previous studies (Amenta, Günther, \& Marelli, 2020; Günther \& Marelli, 2019, 2020). The CAOSS model represents compound meanings $c$ as $c=M * u+H * v$, so the $n$-dimensional modifier vector $u$ and head vector $v$ are multiplied with a $n \times n$-dimensional weight matrix ( $M$ or $H$ ) before being summed up. Note that the CAOSS model still adds the exact same modifier vector $(M * u)$ to each head noun, the very mechanism which Lake and Murphy (2021) doubt to be able to capture semantic relations between the constituents. These weight matrices $M$ and $H$ are estimated and optimized via training on all existing compounds in the corpus (so that their whole-word vectors are best predicted by the formula $c=M * u+H * v$ ), again relying on least-squares multivariate multiple regression. In the present study, this training set consists of 4,880 compounds (a) from several large- 
Mapping: Training (on all compounds except $C$ )
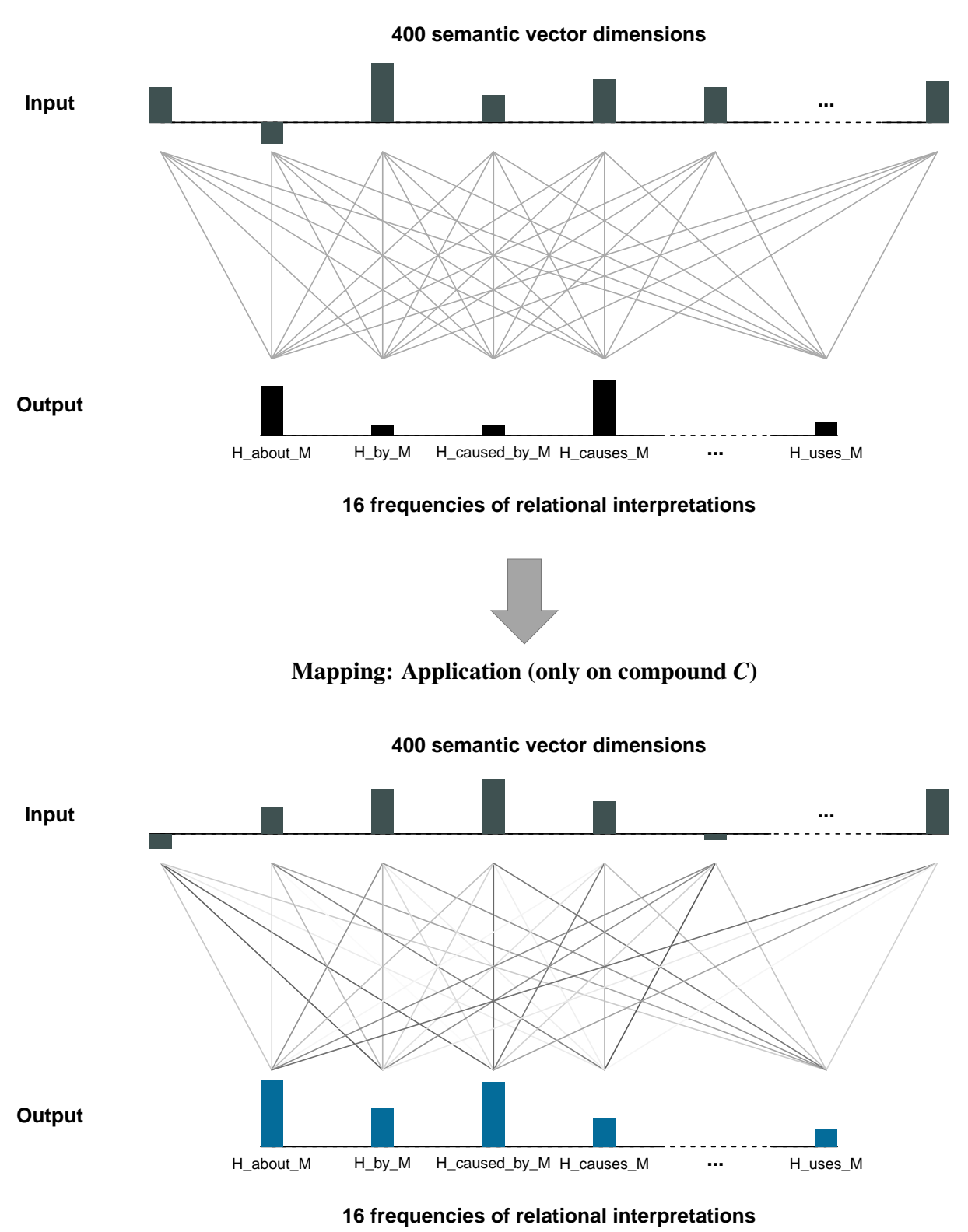

Figure 1. The mapping system, predicting frequencies for the 16 relational interpretations for a compound (output) from the 400 dimensional values of the distributional semantic vector representing the compound meaning (input). The weights (represented as lines) of the mapping system - a least-squares multivariate multiple linear regression model - are trained on all compounds in the data set except for one (upper panel), and then applied to predict the relational interpretations for this left-out compound (lower panel) (LOOCV).

scale studies and databases (Gagné et al., 2019; Günther \& Marelli, 2019; Juhasz, Lai, \& Woodcock, 2015; Kim, Yap, $\&$ Goh, 2019) with a frequency of at least 50 in the corpus described above, as well as (b) all compounds in the dataset analyzed here (Schmidtke, Gagné, Kuperman, Spalding, \& Tucker, 2018).

\section{Training the mapping system}

In the previous section, we described three methods to model speakers' representations of the compounds presented to them - that is, three vector representations (whole-word, additive model, CAOSS) for the left-out test item from which 
the relational interpretations could be predicted. In the same way, we can use these three different vector representations in the training step of our mapping system (see Figure 1). To establish reference points that allow us to judge the performance of these three different mapping systems, we additionally employed several baselines for training.

In the completely random baseline, the semantic vector for each compound in the training set is replaced with a randomly-sampled semantic vector from the semantic space by Baroni, Dinu, and Kruszewski (2014). All variance in relational judgments predicted by this baseline can be attributed to a general bias for certain relations in the dataset: For example, assume that $50 \%$ of participants always selected head FOR modifier, 25\% always selected head MADE OF modifier, and the final $25 \%$ always selected head HAS modifier, irrespective of the compound presented to them. This response pattern could be perfectly predicted also without any actual information encoded in the semantic vectors, and even a regression trained on completely random vectors could perfectly predict the correct relations via its intercepts alone (by setting all regression weights to zero, see Figure 1).

In the random whole-word baseline, the semantic vector for each compound in the training set is replaced with the whole-word vector for another randomly-sampled compound from the dataset by Schmidtke, Gagné, Kuperman, Spalding, and Tucker (2018). Any potential increase in performance for this baseline in comparison to the completely random baseline can be attributed to some non-specific relational information captured by all semantic vectors for compounds.

Finally, in the random CAOSS baseline, the semantic vector for each compound in the training set is replaced with the CAOSS vector for another compound in the dataset by Schmidtke, Gagné, Kuperman, Spalding, and Tucker (2018). Any potential increase in performance for this baseline over the random whole-word baseline can be attributed to some non-specific relational information captured by the CAOSS model.

\section{Results}

If the mapping system predicted negative frequencies for relational interpretations, these were set to zero. However, we observe the very same pattern of results (with a slightly lower level of performance for all models) without this adjustment, as reported in Supplementary Material A. ${ }^{2}$

As the measure for model performance, we calculated for each compound the Pearson correlation between the actual distribution of relational judgments and the model estimates. The highest levels of model performance were reached when the left-out test compounds were represented via the CAOSS model, see Figure 2. The detailed numerical values, along with the results for the other two options (test compound represented via the whole-word or additive model) are reported in the Supplementary Material.

We define that one mapping system outperforms another mapping system if there is no overlap between their respective .95-confidence intervals (CIs). As can be seen in Figure 2, the mapping systems trained on the whole-word vectors and CAOSS vectors outperform all baselines, with no overlap between the CIs of the best-performing baseline (random whole-word) and these two mapping systems. The performance of the mapping system trained on whole-word vectors is numerically higher than of the mapping system trained on CAOSS vectors, but it does not clearly outperform this setup. Examples for very good and very poor predictions with thus setup are presented in Figure 3.

\section{Discussion}

In this first study, we find that distributions of relational interpretations for compounds can indeed be predicted well above chance level from their semantic vector representations, which demonstrates that the relevant information needs to be encoded in these vectors to begin with. In the bestperforming setups of the mapping system, the left-out test compounds are represented via their CAOSS vectors (Marelli et al., 2017), and the mapping system is trained either on the whole-word vectors or the CAOSS vectors of the remaining 574 compounds (Figure 2). The performance of these two setups is comparable, with a non-significant numerical advantage for training on the whole-word vectors. During the training phase, the mapping system can thus learn the relevant relational information from these compound representations.

At first glance, it might appear surprising that the performance of these setups is better than for setups in which the left-out test compounds are represented via wholeword vectors (representing the lexicalized meaning of these compounds; Marelli, Dinu, Zamparelli, \& Baroni, 2015), given that the item set only consists of familiar compounds (see Supplementary Material A). However, note again that Schmidtke, Gagné, Kuperman, Spalding, and Tucker (2018) explicitly instructed their participants to pretend that they were learners of English who have never seen the specific combination of words before, which encourages compositional processing and more "literal" interpretations that deviate from the actual, lexicalized meanings of some words. This might in turn lead to increased performance of a compositional model such as CAOSS as opposed to other possible instructions that focus more on lexicalized meanings. Moreover, compounds were presented in their open form (i.e. with constituents separated, crow bar), rather than their closed one

\footnotetext{
${ }^{2}$ Another variant in which we used logit-transformed relative frequencies, which should in principle provide more adequate data for a linear regression model, performed worse than the models reported here.
} 


\section{Experiment 1}

\section{Test compound represented via CAOSS}

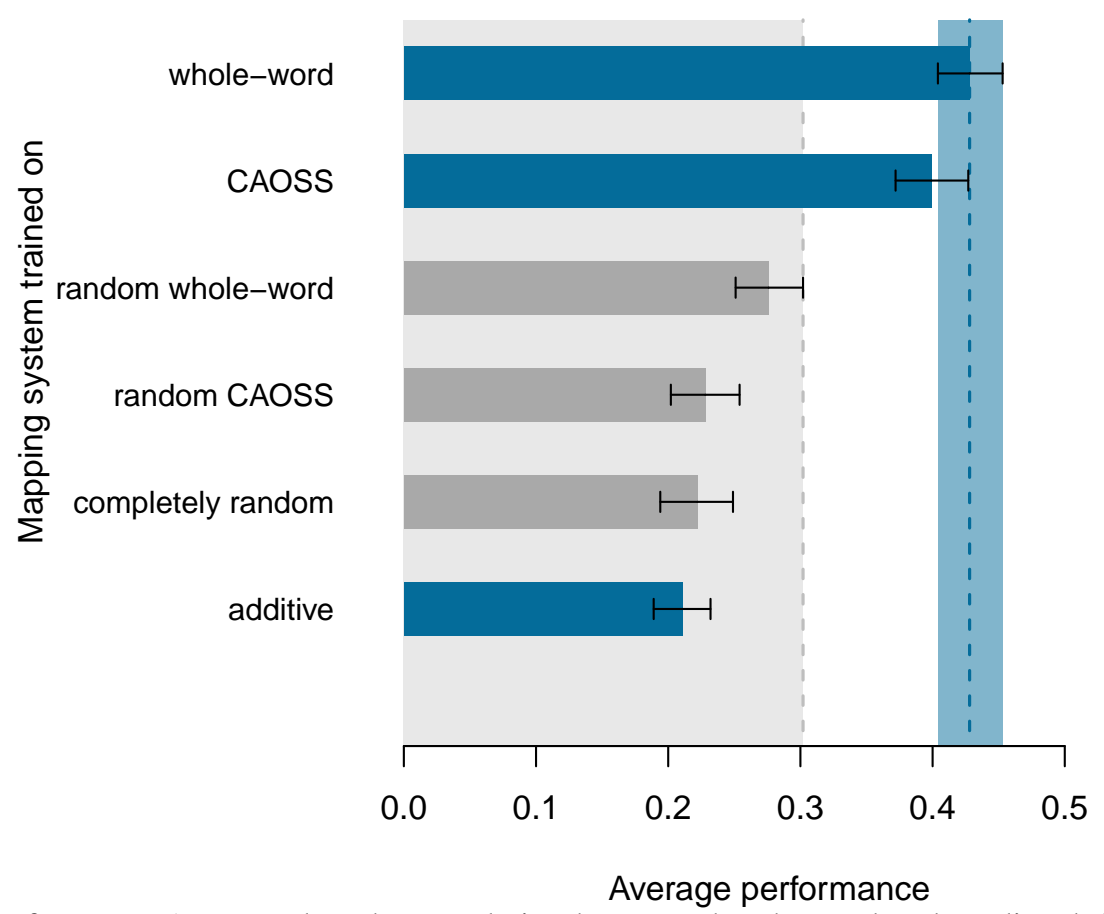

Figure 2. Average performance (measured as the correlation between the observed and predicted distributions of relational judgments) with .95-confidence intervals for all mapping systems in Experiment 1. Cases where the mapping system was trained on vectors representing compound meanings are displayed in blue, all baselines are displayed in gray.

(i.e., as a unique string, crowbar). This may further drive participant's judgments towards actively-computed compositional meanings, since open compound forms are especially known to elicit such automatic combination operations in compound processing (Ji et al., 2011; Marelli et al., 2015).

Thus, it may be the case that the relational interpretations collected by Schmidtke, Gagné, Kuperman, Spalding, and Tucker (2018) are fully compositional ones, and we cannot entirely exclude that we effectively predicted the meanings of novel compounds in Experiment 1. However, this is very unlikely: It requires participants to a) always be able to follow to the letter the provided instructions, even in difficult scenarios like this when they are asked to pretend they don't know a (arguably very familiar) term, and b) to completely abstract from their acquired meaning representations for the combinations presented to them for their decision. It is more plausible that these whole-word representations influence participant judgments at least to some degree (which is also why we considered these whole-word representations in our mapping system).

The fact that even the performance of the random baseline models is above zero indicates that there is some systematic bias in the distribution of relations, which our regression-based mapping system can pick up in its intercepts even without informative predictors. However, using actual dimensional values of semantic vectors as predictors clearly adds relevant semantic information, over and above these base rate probabilities, that the mapping system can pick up and utilize to make more accurate predictions for individual compounds. Importantly, due to the LOOCV approach (Richie et al., 2019; Utsumi, 2020; Zou \& Bhatia, 2021), each individual test compound is not included in its respective mapping system's training set, thus minimizing the risk of overfitting.

In this first experiment, we only considered relational interpretations of familiar compounds. However, the argument by Lake and Murphy (2021) concerning the interpretability of conceptual combinations was not directed at familiar compounds (although it can be applied to these cases too), but at novel conceptual combinations like "apartment dog". The results of our first experiment do not necessarily imply that relational interpretations can also be predicted from semantic vectors for entirely novel compounds: On the one hand, it might be the case that the relational pat- 
good predictions $(r>.9)$

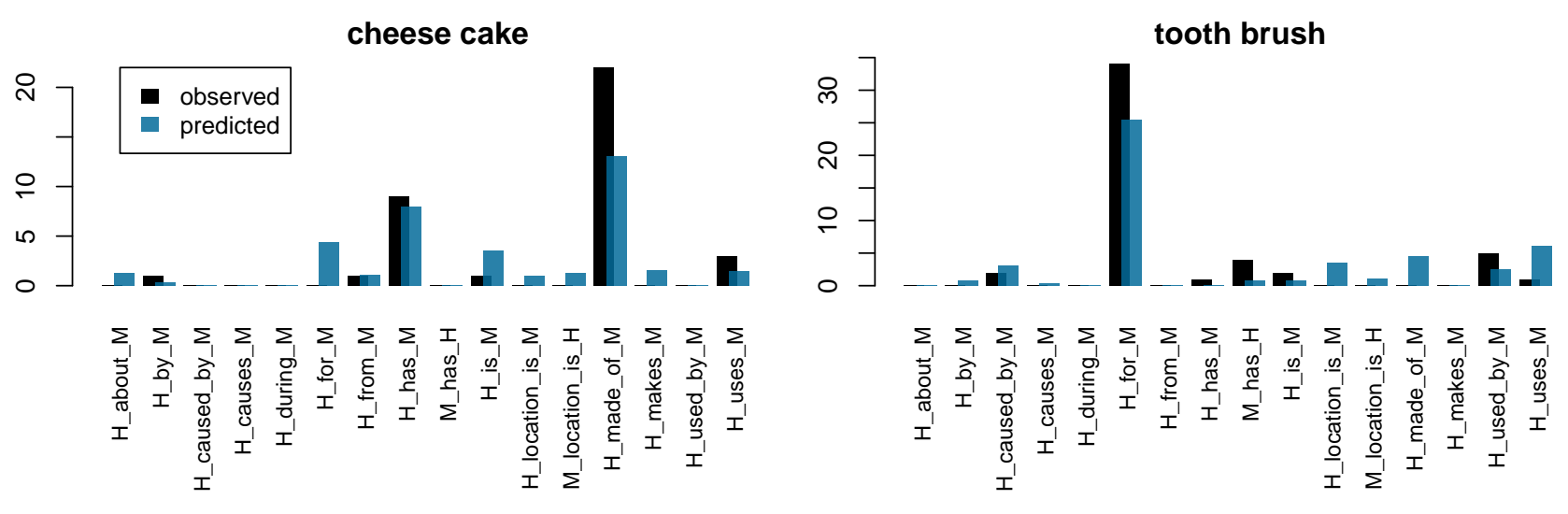

poor predictions $(r<.1)$
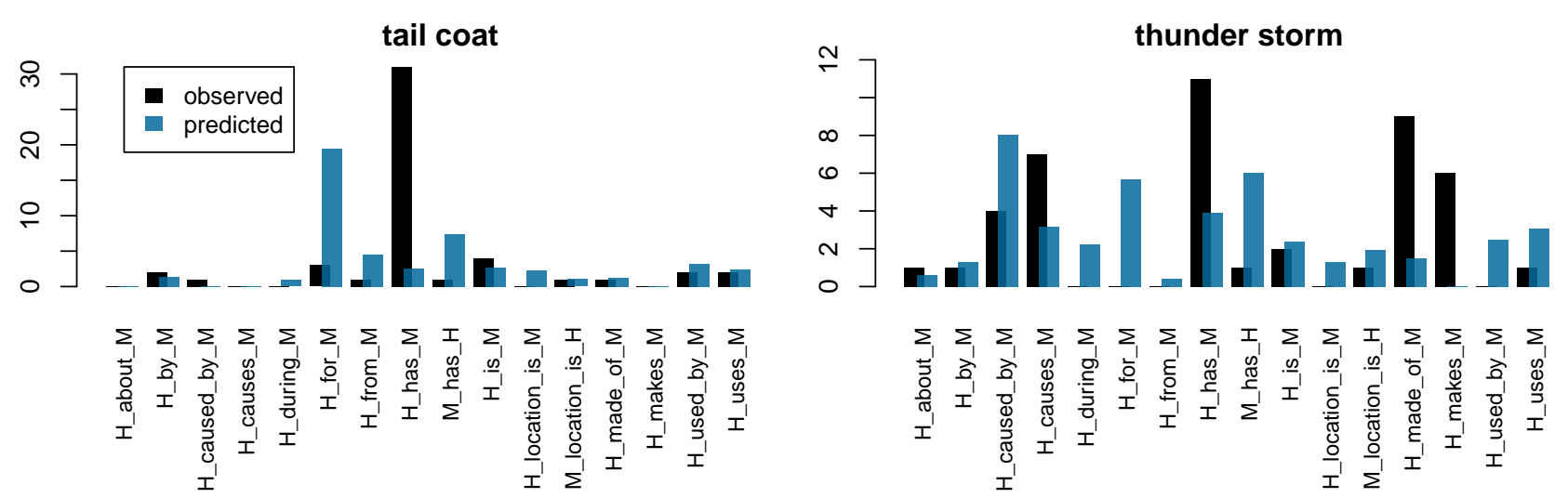

Figure 3. Good and poor predictions of the best-performing mapping system (trained on whole-word vectors, with test vectors represented via CAOSS) in Experiment 1. The y-axis indicates the observed and predicted frequencies of the respective interpretations.

terns of familiar compounds are systematically different from those of novel compounds. On the other hand, only familiar compounds have a conventionalized, lexicalized meaning which may influence judgments; in contrast, interpretations of previously-unseen compounds and their semantic relations are heavily underspecified when compared to existing compounds (Ryder, 1994; Schäfer \& Bell, 2020), which should lead to a much higher variability in interpretations. In the second study, we thus investigate whether relational interpretations can be predicted on the basis of the (subsymbolic) dimensions of semantic vectors even for completely novel compounds.

\section{Experiment 2: Novel compounds}

In order to investigate whether relational interpretations of novel compounds (which participants are not supposed to have encountered before) can be predicted from vectors representing their meaning, we collected a new dataset of such interpretations for a set of 408 novel compounds. To test the generalizability and the limits of these predictions, half of these compounds shared at least one constituent with a familiar compound in Experiment 1, while the other half did not.

\section{Method}

Participants. 400 monolingual English-speaking participants took part in the experiment via the crowdsourcing platform Prolific.co. Two additional participants were excluded because they indicated a native language different from English. 251 participants self-identified as female, 141 as male, and 8 indicated another gender or provided no response. The participants' age ranged from 18 to 88 years ( $M=35.0$ years, $S D=13.5$ years). Of these 400 participants, 34 had to be excluded because they were deemed noncompliant on the basis of their responses, as described below. Participants received $£ 2.50$ for their participation in the study. 
Material. The item material of this study consisted of 408 open-form novel compounds such as mouse dog or wood brush. All of these compounds were obtained from the item set used in the reaction time studies by Günther and Marelli (2020). All items in this set were initially selected by Günther and Marelli (2020) because they were not attested (neither in open nor closed form) in the same 2.8-billion word corpus used for model-training purposes in the present paper (see the Method section of Experiment 1). For 204 of these compounds, at least one of their constituents appeared in the item material employed in Experiment 1 (Schmidtke, Gagné, Kuperman, Spalding, \& Tucker, 2018), although not necessarily in the same position. The other 204 compounds consisted of constituents that did not appear in any position in this latter item set. These 408 items were randomly arranged into eight experimental lists of 51 items, which were presented to 50 participants each (resulting in the same number of judgments per item of Schmidtke, Gagné, Kuperman, Spalding, \& Tucker, 2018).

In addition, each list contained four catch trials aimed at identifying non-compliant participants. These were selected from the items with the lowest entropy (i.e., highest agreement) in the Schmidtke, Gagné, Kuperman, Spalding, and Tucker (2018) dataset: snow man (man MADE OF snow), stone ware (ware MADE OF stone), sweat band (band FOR sweat), and tea time (time FOR tea). Apart from these normatively correct responses, we also considered as "correct by convention" responses which were selected by at least 10 participants (for example, in our data the responses man FROM snow and man IS snow were given 61 and 18 times, respectively). A participant was considered non-compliant if fewer than 3 out of 4 of their responses to the catch trials were classified as correct, which was the case for 34 participants.

Procedure. After informed consent, participants were presented with a page explaining the 16 different possible compound relations with examples (Schmidtke, Gagné, Kuperman, Spalding, \& Tucker, 2018), which they could also open in a new tab as a cheat sheet during the actual study. Participants were then instructed to select an interpretation for each of the 55 expressions presented to them, by choosing the most adequate of the possible relations connecting the two constituents.

In each trial, the question "What does [modifier head] mean? was presented in bold letters on the top of a page, and participants selected their preferred relation using radio buttons labeled with the 16 possible interpretations, specifically adapted to the compound presented (e.g. brush FOR wood or brush MADE OF wood if the compound was wood brush).

For each participant, the 55 compounds were presented in random order. Most participants took between 10 and 30 minutes to complete the study (median $=16.8 \mathrm{~min}$ - utes).

\section{Results}

After exclusion of non-compliant participants, the dataset of Experiment 2 contained between 44 and 50 responses per compound. As expected, the relational entropy (as defined by Schmidtke, Gagné, Kuperman, Spalding, \& Tucker, 2018; Schmidtke et al., 2016) of the 408 novel compounds in Experiment 2 was higher on average than for the 575 familiar compounds in Experiment $1\left(M_{2}=2.338\right.$ vs $M_{1}=2.131$, $t(797)=-4.97, p<.001)$, indicating higher interpretational variability in novel compounds as compared to existing compounds.

All items in this dataset are novel compounds that do not appear in our source corpus; whole-word semantic vectors representing their meanings are hence not available. Therefore, we can only consider compositional semantic vectors for these compounds (obtained via CAOSS or the additive model) to represent the meanings of these test compounds.

We used the same mapping systems trained in Experiment 1 - that is, the mapping systems trained on familiar compounds - to predict the relational frequency distributions for all 408 novel compounds. In this setup, the mapping system always extrapolates interpretations for novel compounds from the familiar compounds it has encountered before; thus, we do not assume a separate interpretational system for novel compounds. Since all these novel compounds forming the test set of Experiment 2 are outside the training set of these mapping systems, we do not need to employ an LOOCV approach in Experiment 2 - the mapping systems can be trained on all 575 existing compounds examined in Experiment 1, and then applied to predict relational distributions for the novel compounds. We trained the mapping system on the same semantic models (whole-word, CAOSS, additive) and baselines (random whole-word, random CAOSS, completely random) as in Experiment 1, and again measure model performance as the mean correlation between the observed and predicted relational judgment distribution.

As in Experiment 1, the highest levels of performance were reached when the test set compounds (i.e., the novel compounds) were represented via the CAOSS model. The results for this setup are presented in Figure 4, with exact numerical values (as well as the results for the setup where the test set compounds are represented via the additive model) presented in the Supplementary Material B. As can be seen, the pattern of results is very similar to Experiment 1: Model performance is highest when the mapping system was trained on whole-word or CAOSS vectors, with very similar levels of performance for these two setups. Both setups clearly outperform the best baseline (the random whole-word model, which also was the best baseline in Experiment 1).

In a next step, we conducted separate analyses for 


\section{Experiment 2}

\section{Test compound represented via CAOSS}

\section{all compounds}

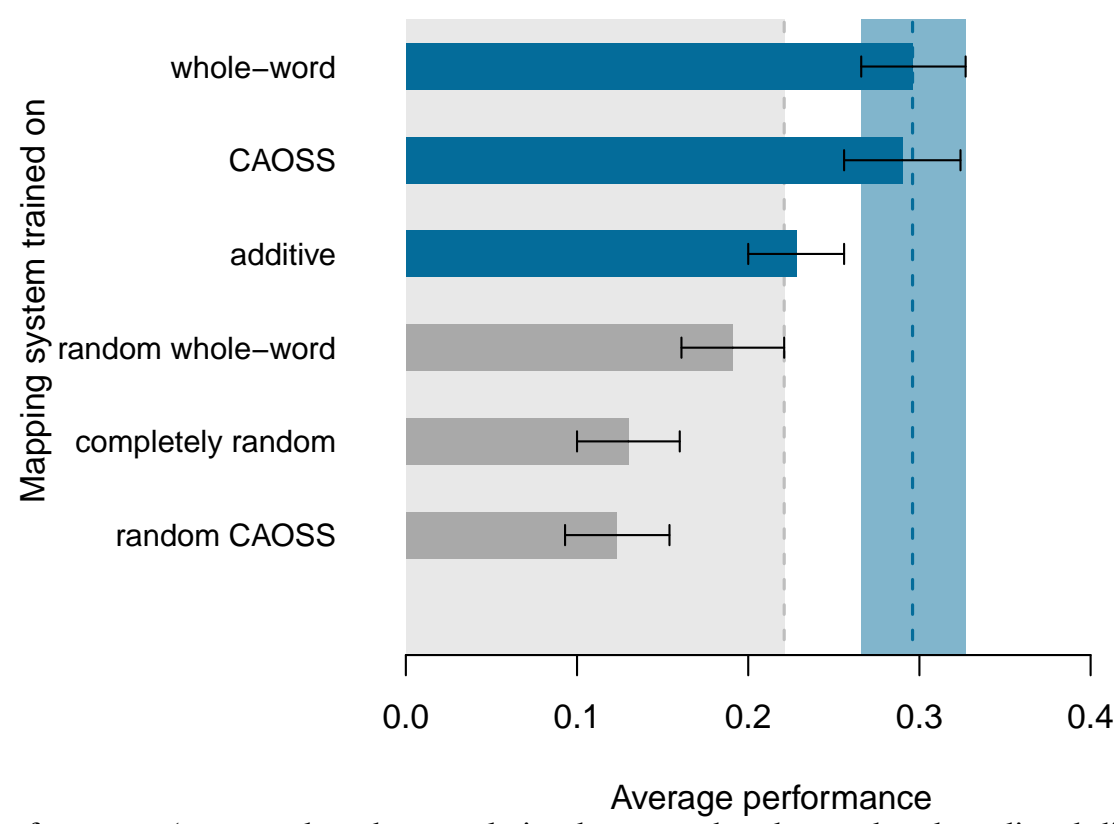

Figure 4. Average performance (measured as the correlation between the observed and predicted distributions of relational judgments) with .95-confidence intervals for all baseline models (in gray) and all semantic models (in blue) in Experiment 2, when the novel compounds are represented via the CAOSS model. Model refers to the model on which the mapping system is trained.

the 204 novel compounds that shared at least one constituent with the 575 existing compounds from Experiment 1 and the 204 compounds that shared no constituent with this set. The results of these analyses are reported in Figure 5. As can be seen, model performance was considerably higher in the shared-constituent subset. In the shared-constituent subset, the setups in which the mapping systems were trained on CAOSS vectors or whole-word vectors outperformed all baselines, with no clear difference in the performance between these two models. In the no-shared-constituent subset, the confidence interval the setup in which the mapping system was trained on whole-word vectors shares only a residual overlap with that of the random whole-word baseline. For the interpretation of these results, note that the confidence intervals in the subset analyses are generally wider than in the complete-set analyses, because they are based on only half of the items. Good and poor predictions for novel compounds from both subsets (for the setup in which the mapping system was trained on whole-word vectors) are presented in Figure 6.

\section{Discussion}

Overall, frequency distributions of relational interpretations for novel compounds can successfully be predicted from semantic vectors representing their meanings, as demonstrated by the finding that mapping systems employing semantic models outperform random baselines. This demonstrates that semantic vectors, obtained through an adequate compositional model, indeed encode possible interpretations of novel compounds and, most importantly, their variability across speakers.

With our set of models, the overall performance is somewhat lower for novel compounds as compared to familiar compounds, which might be expected given that the mapping system is trained on familiar compounds. However, a closer look at the results reveals a more nuanced pattern: For novel compounds that share at least one constituent with the mapping system's training set, predictions are actually on par with those for familiar compounds in Experiment 1 . The mapping system only begins to struggle for compounds that share no constituent with this training set in this difficult case, where potential relations need to be es- 


\section{Experiment 2: subset analysis}

Test compound represented via CAOSS

shared const.

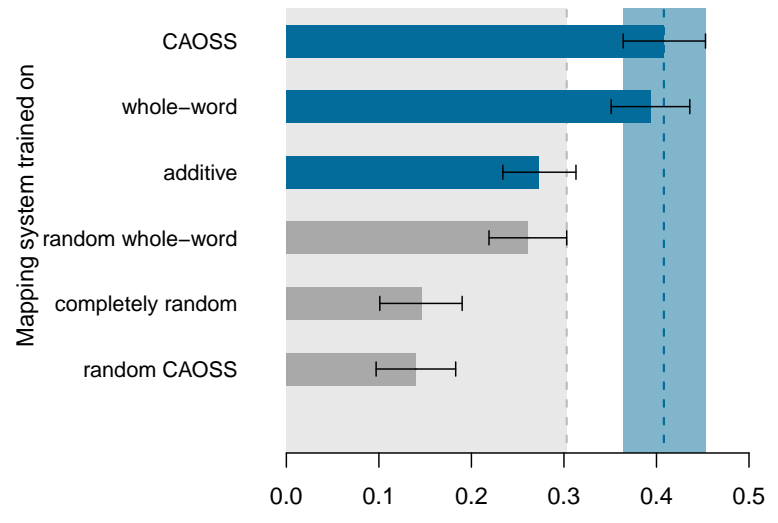

Average performance
Test compound represented via CAOSS

no shared const.

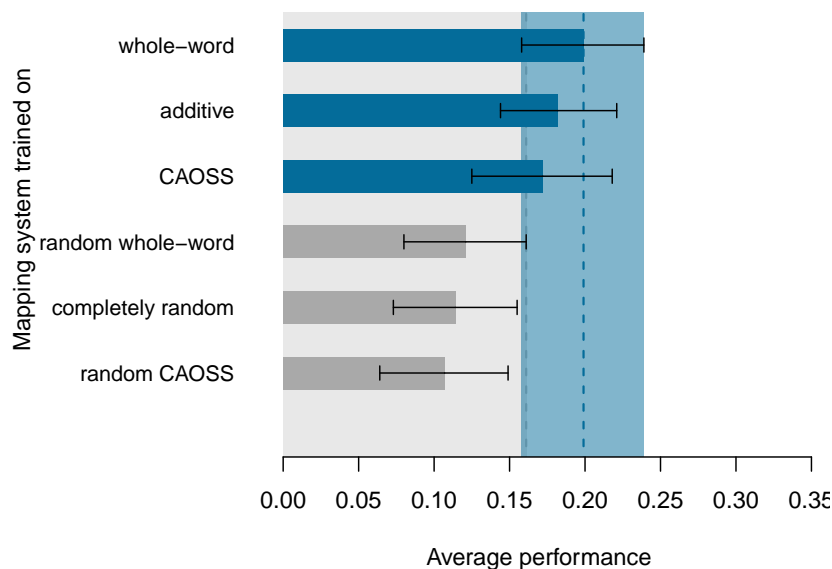

Figure 5. Average performance (measured as the correlation between the observed and predicted distributions of relational judgments) with .95-confidence intervals for all baseline models (in gray) and all semantic models (in blue) in the subset analysis of Experiment 2, when the novel compounds are represented via the CAOSS model. Model refers to the model on which the mapping system is trained.

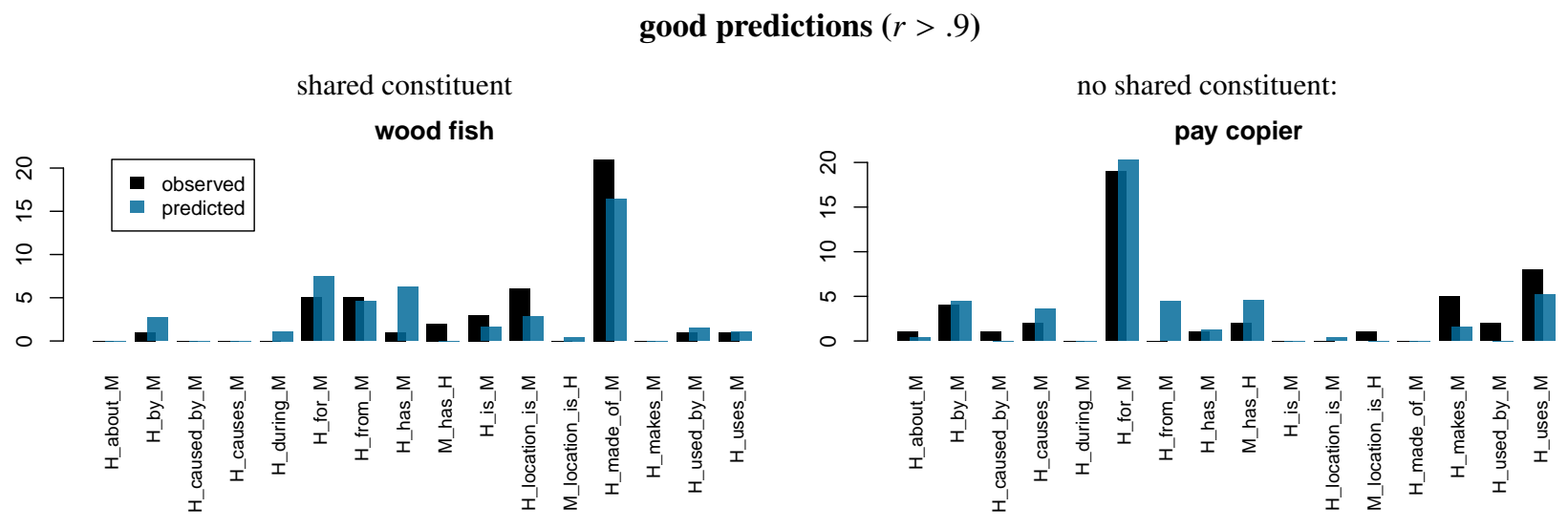

poor predictions $(r<.1)$

shared constituent

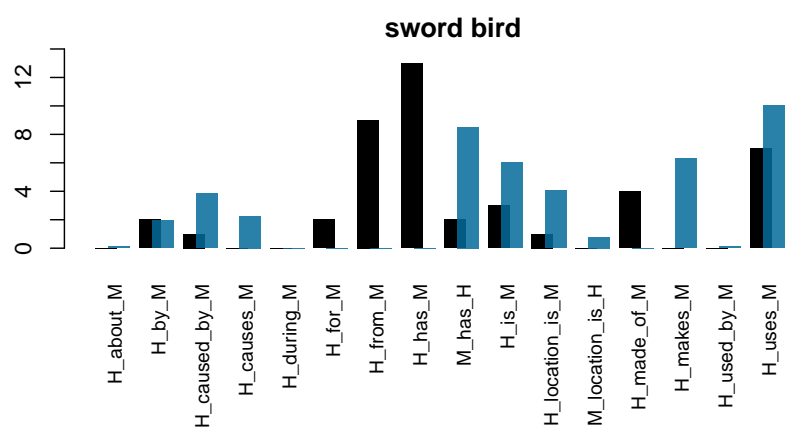

no shared constituent:

alder mind

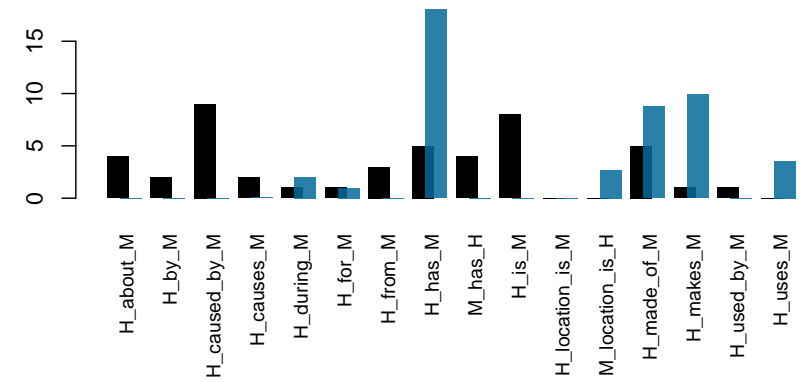

Figure 6. Good and poor predictions of the mapping system trained on CAOSS compound vectors in Experiment 2. The y-axis indicates the observed and predicted frequencies of the respective interpretations. 
timated purely from the isolated meanings of the compound constituents without any direct experience of their relational behavior in compounds, semantic models obtain numerically higher levels of performance than random baselines, but do not fully outperform these baselines (which can potentially be attributed to the smaller item sets in the subset analyses).

It is important to note that this limitation is at least partly a methodological one, rather than a genuine generallevel issue: The training set of our mapping system consisted of the 575 familiar compounds included in Experiment 1, along with their 535 different constituents. This is only a fraction of all existing English compounds - the LADEC database (Gagné et al., 2019) alone contains 8,376 compounds consisting of 3,774 constituents. In regular language use, most compounds are part of productive morphological families, and most novel compounds which are actually produced by speakers share at least one constituent with an existing compound (Libben, 2014). Hence our results (where the non-shared subset makes up half of the entire novel compound set) can be considered an especially conservative estimate of the mapping system's predictive power.

\section{General Discussion}

In the present article, we tested whether distributions of relational interpretations for compounds, as produced by groups of native speakers, can be predicted from their corresponding vector representations, induced from samples of language usage. In the first study, we show that this is indeed possible for relational interpretations for familiar compounds (Schmidtke, Gagné, Kuperman, Spalding, \& Tucker, 2018), using an LOOCV regression approach. In the second study, we demonstrate that this mapping system can also be successfully employed to predict relational interpretations for novel compounds, even at a comparable level of performance if these contain familiar constituents in unfamiliar positions or unfamiliar constituents. The mapping system only starts to struggle for novel compounds consisting of two entirely unfamiliar constituents. These results show that semantic vectors can indeed stand up to the fundamental theoretical challenges directed at them (Glenberg \& Robertson, 2000; Lake \& Murphy, 2021): They encode interpretable information, and they capture variability in meaning (for further discussions, see also Günther et al., 2019).

The present results suggest that the CAOSS model (Marelli et al., 2017) produces adequate semantic vectors to represent (novel) compound meanings. Note that our results clearly warrant the employment of a compositional model such as CAOSS, even though it is obviously more complex than simply relying on whole-word vectors and introduces additional degrees of freedom. In Experiment 1, setups where the test compounds are represented via the CAOSS model clearly outperform those where test compounds are represented via the whole-word model (as described in the Results section of Experiment 1; compare also Table 2 with Table 3 in the Supplementary Materials). This is even clearer in Experiment 2, where a compositional model needs to be employed to represent the test compounds simply because no wholeword vectors are, by definition, available for them. Thus, in both experiments, reaching the levels of empirical performance reported here requires a compositional model.

This does not imply that the CAOSS model specifically needs to serve as this compositional model. Taking all our results into account it generally outperforms the Additive model (which serves as a generic baseline), but there may still be better options. However, we want to point out that the purpose of the current study was not to provide an engineering solution, meticulously exploring constellations of model parameters that result in optimal performance; rather, we wanted to demonstrate in a proof of principle that even an off-the-shelf model without task-specific fine-tuning is able to successfully predict relational judgments. This statement holds for the compositional model as well as the linear regression model that serves as our mapping system, which both could surely be replaced by more sophisticated and finetuned solutions.

\section{Interpretability}

Concerning the issue of interpretability, Lake and Murphy (2021) criticized as the main shortcoming of semantic vector representations for novel combinations that "there is no easy way to find out how the model is interpreting "apartment dog" " (p. 31). Our results (especially those of Experiment 2) clearly refute this intuition: Of course, such qualitative interpretations require additional steps over simply inspecting the vector dimensions at face value, and hence a direct reading is not possible for a human observer. This is analogous to other codes, like Morse code, telegraphy, or radio waves, which all require a decoding system in order to transform the encoded information into a format understandable for humans. Nevertheless, the information is clearly encoded in the signal. In our case, the decoding role is played by our mapping system, a linear regression translating a semantic vector into an understandable format. Even though this is a very simple approach, it reaches respectable performances in the present experiments. Of course, one could employ more sophisticated architectures which can further improve the performance of the mapping system, such as multi-layer neural networks or more complex non-linear models. However, our results already demonstrate that meticulous parametrization and fine-tuning are, in principle, not necessary for this mapping to work (see also Günther, Petilli, Vergallito, \& Marelli, 2020, for similar arguments). This implies that the required information is encoded in the semantic vectors forming the input of the mapping system.

Of course, semantic relations between constituents are only one type of qualitative information, and one that 
is relatively specific to compounds. As described in the Introduction, the (relatively) closed set of possible relational interpretations renders these words an ideal test case for investigating the issue of interpretability. However, this does not imply that we can maybe salvage compounds, while semantic vectors for all other words remain uninterpretable: Recently, a large number of studies (for an overview, see Snefjella \& Blank, 2020) have demonstrated that a range of other types of qualitatively-intepretable information, such as semantic features (Hollis \& Westbury, 2016; Hollis, Westbury, \& Lefsrud, 2017; Sommerauer \& Fokkens, 2018), sensorimotor information (Utsumi, 2020), visual features (Günther et al., 2020), emotional information (MartínezHuertas, Jorge-Botana, Luzón, \& Olmos, 2021) or numerical estimates (Zou \& Bhatia, 2021) can be predicted from semantic vectors using very similar regression approaches to the one employed in the present study. Thus, semantic vectors implicitly encode a large variety of human-interpretable information - it just needs to be decoded, which can be successfully achieved by means as simple as a linear regression.

Notably, these semantic vectors were not explicitly constructed with the aim of encoding these interpretations: Similar to all standard distributional models, the training objective of the cbow model is simply to predict words from other words in their immediate surroundings (Mikolov et al., 2013), and the specific instance of the model employed in the present study was evaluated mostly against similarity judgments and categorization tasks for individual words (Baroni, Dinu, \& Kruszewski, 2014). Similarly, the training objective of the CAOSS model was only to predict compound vectors from their constituent vectors. Thus, the encoding of relational interpretations - and the other dimensions cited in the previous paragraph (Snefjella \& Blank, 2020) - is an emergent property of these semantic vectors, a byproduct of harvesting learning experience (which aligns with results by Schmidtke, Gagné, Kuperman, \& Spalding, 2018 showing that individuals can extract such relational knowledge from written language experience).

The present studies were designed to show that relational information can be decoded from patterns of language usage as captured by distributional models, but until further empirical studies are available we can only speculate about how this information ends up encoded in linguistic distributions to begin with. For other semantic properties, the case appears to be much clearer: For example, the emotional valence of words is influenced by their direct linguistic context (reading "Their sweet flower girl was sitting in the magical garden. She picked a wORD and smelled it." will result in a positive connotation for wORD, while "Their ill flower girl was sitting in the rotten fungus. She picked a wORD and smelled it." will result in a negative connotation; Snefjella, Lana, \& Kuperman, 2020), which enables distributional models to capture affective information (Hollis et al., 2017; Martínez-Huertas et al., 2021). At first glance, such context effects are arguably less obvious for relational interpretations: what would be the systematic usage pattern for "H LOCATION IS M"-compounds? However, a possible answer is already suggested by Gagné and Shoben (1997) in their seminal introduction of the CARIN theory, with the postulation that specific constituents (and especially specific modifiers) tend to occur with specific relations (an assumption which has since then received substantial empirical support; Gagné \& Spalding, 2013): For example, for compounds including the modifier "mountain", the "H LOCATION IS mountain"-interpretation is far more likely than, say, a "H DURING mountain"-interpretation. Relational interpretations of compounds are thus linked to the lexical semantics of their constituents. In turn, these constituent semantics heavily influence the semantics of the compounds embedding them (at least for the far more numerous transparent compounds; Gagné et al., 2019). Since, according to the distributional hypothesis, lexical semantics determine patterns of language use (Lenci, 2008), compounds with different relational interpretations should be used in somewhat different linguistic contexts. However, we expect relational impact to be relatively subtle, which is reflected in the present empirical results: our predictions of relational interpretations are quantitatively less successful than, for example, the extrapolation of emotional valence from distributional vectors (Hollis et al., 2017; Martínez-Huertas et al., 2021). Note that this argument so far focused entirely on the whole-word meaning of compounds; once we consider compositional meanings, the influence of the individual consituent meanings will become only more relevant, as discussed in the Novel combinations section later on.

\section{Variation in meaning}

A similar argument applies to variation in meaning, which (as demonstrated in the present study) can also be decoded from semantic vectors. This may sound counterintuitive if, as it often happens, one assumes that a single vector representation for a word should correspond to a single symbolic meaning (see Günther et al., 2019). However, a semantic vector can be taken as a pattern of activation indicating the relative likelihood of different interpretations for its associated word (Dinu \& Lapata, 2010; Marelli \& Baroni, 2015). Indeed, the very same principle can be directly observed in the distributions of relational interpretations (Schmidtke et al., 2016; Schmidtke, Gagné, Kuperman, Spalding, \& Tucker, 2018), which themselves can be conceptualized as multidimensional vector representations (see Table 1 as well as Figure 1). As shown in both studies reported here, this distribution of relational interpretations for compounds is not just a random example of another meaning-related vector format - it can be directly predicted from the compounds' semantic vectors (compare Figure 3 and Figure 6). 
In this context, it must be remarked that the variation in meaning we considered was observed between participants, since each participant only provided a single relational interpretation for each compound. Thus, a single semantic vector for each compound can capture inter-individual differences in its interpretation. This can be explained by considering that the semantic vectors were not trained on the individualized language experience of a single speaker, but instead on a large corpus that serves as a proxy for the language experience of a speaker population, resulting in an aggregate semantic representation at the population level (Günther et al., 2019; Johns, Jones, \& Mewhort, 2016). It is up to future studies to investigate to what extent semantic vectors can predict meaning variation within individual speakers (for example, by lifting the restriction that each participant can only select one relational interpretation per compound, see Schmidtke, Gagné, Kuperman, \& Spalding, 2018).

\section{Novel combinations}

The first study presented here demonstrates that semantic vectors predict interpretations of existing compounds above chance level. In this study, we observed that the best predictions are obtained from a compositional model that actively productively combines the meanings of the two constituents (CAOSS; Marelli et al., 2017). As noted in the discussion of this experiment, this may however be a consequence of the specific instructions by Schmidtke, Gagné, Kuperman, Spalding, and Tucker (2018), who asked their participants to imagine they were non-native speakers of English who encountered an expression for the first time (i.e., to treat the compounds as if they were novel combinations). However, the fact that the relational entropy characterizing these rating distributions predicts on-line processing times for the same compounds (Schmidtke, Gagné, Kuperman, Spalding, \& Tucker, 2018; Schmidtke et al., 2016) suggests that such compositional representations indeed reflect a psychological reality even for familiar compounds.

Experiment 1 already gives a first indication that semantic vectors - when equipped with adequate compositional models such as the CAOSS model (Marelli et al., 2017) - can capture the meanings of novel combinations. However, more compelling evidence for the capability of semantic vectors to represent the meanings of novel combinations comes from Experiment 2, where relational interpretations of such novel combinations could be predicted from their semantic vectors. While the performance was much higher for compounds that shared at least one constituent with the training set of the regression model, where it reached the level of existing compounds, this was also to some extent the case for compounds consisting only of constituents that the regression model had never encountered before (see Table 6). Critically, because all these novel combinations those sharing and those not sharing a constituent - are by def- inition unattested in the source corpus, their semantic vectors needed to be derived by using a compositional model such as the CAOSS model.

The present study extends upon previous research which showed that CAOSS is well-suited to represent compositional compound meanings, accounting for various empirical phenomena related to conceptual combination and compositionality. For example, Marelli et al. (2017) showed that CAOSS captures the relational priming and relational dominance effects observed in the literature (Gagné, 2001; Gagné \& Shoben, 1997). Furthermore, the model has been employed to detect automatic meaning-combination effects during the processing of novel compounds (Günther \& Marelli, 2020), and to demonstrate that semantic transparency effects during the processing of familiar compounds should also be attributed to such active meaningcomposition processes (Günther \& Marelli, 2019). It even allows us to empirically investigate and account for phenomena that have been notoriously hard to capture using other approaches, such as the systematic semantic shift experienced by some words when they are used as compound constituents (Günther \& Marelli, 2021), or a language-invariant mechanism of compounding by being able to partially predict compound meanings in one language from a CAOSS model trained on another language (Günther \& Marelli, 2018). Taken together, these studies also show that compositional distributional models have been evaluated using a wider range of methods and data than just by comparing human judgments to distributional similarities, which is the only class of phenomena that Lake and Murphy (2021) picked out when reviewing the current empirical status of these models.

To this, the present results add that the potential relational pattern related to a given compound naturally emerges from the compositional operation implemented in CAOSS. The evidence from Marelli et al. (2017) suggests this to largely depend on the interplay between the semantic properties of the modifier and the re-weighting process represented by the matrix $M$ : This latter will enhance the features that are most informative for the definition of the relational structure. Thus, the semantic dynamics emerging from CAOSS on the one hand well capture a number of relational phenomena described in the conceptual combination literature (Gagné \& Shoben, 1997; Gagné, 2001), and on the other hand computationally reflect the theoretical frameworks proposed to explain such effects (in particular the CARIN and RICE models; Gagné \& Shoben, 1997; Spalding et al., 2010).

Of course, whereas models like CARIN and RICE are characterized in terms of manipulation of explicit, wellinterpretable symbols, CAOSS (and distributional semantics in general) is founded on distributed representations and nuanced operations aimed at modulating their subsymbolic di- 
mensions. This should not be considered a shortcoming, insofar it will reflect the inherent vagueness of semantic processes and intuitions of human speakers: As demonstrated in the present paper, the degree of disagreement in semantic interpretation by human participants is substantial, even when considering familiar words. Remarkably, the same consideration applies when relying on expert annotations: there is a vast disagreement on which relational taxonomy should be considered the more apt, to the point that it has been claimed that relations are potentially infinite and trying to define a clear-cut set of them is a fruitless endeavor (Downing, 1977; Kay \& Zimmer, 1976). This difficulty in constraining our semantic intuitions at both the layman and the expert level speaks for the necessity of adopting probabilistic, nuanced approaches when attempting to model semantic representations. However, it also poses an important scientific question: if semantic representations are so vague and under-specified, how do we coerce them into declarative boundaries for the purpose of expressing our intuitions? The present results provide a first computational evidence to answer this question, by showing that mapping distributed semantic representations into an array of possible symbolic interpretations does capture the between-speaker disagreement in intuitions about compound-word meanings, in line with computationalneuroscience proposals that see symbols as emergent properties from distributed activations (Eliasmith et al., 2012).

\section{Concluding remarks}

Although the studies presented here demonstrate that qualitative interpretations can be predicted from semantic vectors, we want to express some skepticism and caution towards the view that such qualitative interpretations should be treated as some scientific gold standard that takes epistemological priority over less-intuitive quantitative descriptions. As an example, visible colors are rarely communicated in terms of their actual underlying scientific reality - vectors specifying the wavelength composition of light - but with easilyunderstandable labels such as "red" and "green", discrete simplifications of a complex continuous input that gloss over nuanced differences and variations. The same can be said for qualitative interpretations of linguistic expressions: Interpretations such brush FOR wood and brush MADE OF wood are still severe underspecifications of what a wood brush actually is, and subsume an arbitrary amount of more or less similar entities in a simplified discrete category. Thus, attempting to equate the meaning of wood brush with these discrete interpretations will inevitably fall short of reality.

In this perspective, distributional semantic vectors provide an ideal way to model semantics, because due to their very own structure they do not commit to a specific, well-defined, encapsulated meaning for a given word (Rodd, 2020). Rather, they capture a set of possible (more or less likely) interpretations, each of which can be preferred by a given speaker. One can think about the semantic space containing the semantic vectors as an actual space, where infinite points correspond to infinite meanings. For each word, semantic vectors just indicate the approximate location where appropriate meanings can be found. Each individual speaker, or even the same speaker at different moments, may end up choosing a different interpretation-point. However, in a wellperforming distributional model, all these points will tend to be found in the area defined by the corresponding semantic vector. Distributional semantic models can be in fact considered computational implementations of theoretical proposals like the one by Rodd (2020), that conceptualizes semantic knowledge as a high-dimensional space shaped by experience.

In the present article, we have demonstrated that semantic vectors encode qualitative interpretations as well as their variability across speakers for existing and novel complex expressions in the form of noun compounds, and that this information can be decoded using a simple linear regression approach. Taken together, these findings clearly render the conclusion regarding (compositional) semantic semantic models that "there is no easy way to find out how the model is interpreting "apartment dog." (Lake \& Murphy, 2021 , p. 31) untenable. Recent work has repeatedly demonstrated that information from many different domains can be successfully retrieved from semantic vectors (MartínezHuertas et al., 2021; Richie et al., 2019; Snefjella \& Blank, 2020; Utsumi, 2020; Zou \& Bhatia, 2021), emphasizing the valuable role of computationally well-defined models in investigating the vital role of language experience in building and shaping our conceptual system. These studies speak for the great potential for future research in the field when dropping the (widely-held) implicit assumption that similarities are the only way to probe semantic vector representations (Kintsch, 2001; Lake \& Murphy, 2021), reducing an information-dense representation format to a single metric. Predicting variables on the basis of vector dimensions themselves is an alternative approach that has met with success, and we are certain that yet other methods will provide even further theoretical insights in future studies.

\section{References}

Allen, M. R. (1978). Morphological investigations. (PhD dissertation). University of Connecticut, Storrs, CT.

Amenta, S., Günther, F., \& Marelli, M. (2020). A (distributional) semantic perspective on the processing of morphologically complex words. The Mental Lexicon, 15, 62-78.

Baroni, M., Bernardi, R., \& Zamparelli, R. (2014). Frege in space: A program for compositional distributional semantics. Linguistic Issues in Language Technologies, 9(6), 5-110.

Baroni, M., Dinu, G., \& Kruszewski, G. (2014). Don't count, predict! A systematic comparison of context-counting vs. contextpredicting semantic vectors. In Proceedings of ACL 2014 (52nd 
Annual Meeting of the Association for Computational Linguistics) (pp. 238-247). East Stroudsburg, PA: ACL.

Bell, M. J. (2015). Basic relations and stereotype relations in the semantics of compound nouns. Journal of Cognitive Science, 16, 224-260.

Bell, M. J., \& Schäfer, M. (2013). Semantic transparency: challenges for distributional semantics. In Proceedings of the IWCS 2013 Workshop Towards a Formal Distributional Semantics (pp. 1-10). ACL.

Borghesani, V., \& Piazza, M. (2017). The neuro-cognitive representations of symbols: the case of concrete words. Neuropsychologia, 105, 4-17.

Camacho-Collados, J., \& Pilehvar, T. (2018). From word to sense embeddings: A survey on vector representations of meaning. Journal of Artificial Intelligence Research, 63, 743-788.

Dima, C., \& Hinrichs, E. (2015). Automatic noun compound interpretation using deep neural networks and word embeddings. In Proceedings of the 11th International Conference on Computational Semantics (pp. 173-183).

Dinu, G., \& Lapata, M. (2010). Measuring distributional similarity in context. In Proceedings of the Conference on Empirical Methods in Natural Language Processing (pp. 1162-1172). Cambridge, MA.

Downing, P. (1977). On the creation and use of English compound nouns. Language, 53, 810-842.

El-Bialy, R., Gagné, C. L., \& Spalding, T. L. (2013). Processing of English compounds is sensitive to the constituents' semantic transparency. The Mental Lexicon, 8, 75-95.

Eliasmith, C., Stewart, T. C., Choo, X., Bekolay, T., DeWolf, T., Tang, Y., \& Rasmussen, D. (2012). A large-scale model of the functioning brain. Science, 338(6111), 1202-1205.

French, R. M., \& Labiouse, C. (2002). Four problems with extracting human semantics from large text corpora. In Proceedings of the Twenty-Fourth Annual Conference of the Cognitive Science Society (pp. 316-322). Mahwah, NJ: Erlbaum.

Gagné, C. L. (2000). Relation-based versus property based combinations: A test of the CARIN theory and dual-process theory of conceptual combination. Journal of Memory and Language, 42, 365-389.

Gagné, C. L. (2001). Relation and lexical priming during the interpretation of noun-noun combinations. Journal of Experimental Psychology: Learning, Memory, and Cognition, 27, 236-254.

Gagné, C. L., \& Shoben, E. J. (1997). Influence of thematic relations on the comprehension of modifier-noun combinations. Journal of Experimental Psychology: Learning, Memory, and Cognition, 23, 71-87.

Gagné, C. L., \& Shoben, E. J. (2002). Priming relations in ambiguous noun-noun combinations. Memory $\mathcal{E}$ Cognition, 30, 637-646.

Gagné, C. L., \& Spalding, T. L. (2009). Constituent integration during the processing of compound words: Does it involve the use of relational structures? Journal of Memory and Language, $60,20-35$.

Gagné, C. L., \& Spalding, T. L. (2013). Conceptual composition: The role of relational competition in the comprehension of modifier-noun phrases and noun-noun compounds. In B. H. Ross (Ed.), Psychology of learning and motivation (Vol. 59, pp. 97-130). Elsevier.
Gagné, C. L., Spalding, T. L., Figueredo, L., \& Mullaly, A. C. (2009). Does snow man prime plastic snow?: The effect of constituent position in using relational information during the interpretation of modifier-noun phrases. The Mental Lexicon, 4 , 41-76.

Gagné, C. L., Spalding, T. L., \& Schmidtke, D. (2019). LADEC: Large database of English compounds. Behavior Research Methods, 2152-2179.

Glenberg, A. M., \& Robertson, D. A. (2000). Symbol Grounding and Meaning: A Comparison of High-Dimensional and Embodies Theories of Meaning. Journal of Memory and Language, 43, 379-401.

Günther, F., \& Marelli, M. (2016). Understanding Karma Police: The Perceived Plausibility of Noun Compounds as Predicted by Distributional Models of Semantic Representation. PLOS ONE, 11(10). doi: 10.1371/journal.pone.0163200

Günther, F., \& Marelli, M. (2018). The language-invariant aspect of compounding: Predicting compound meanings across languages. In E. Cabrio, A. Mazzei, \& F. Tamburini (Eds.), Proceedings of the Fifth Italian Conference on Computational Linguistics (CLiC-IT 2018) (pp. 230-234). Turin, Italy: Accademia University Press.

Günther, F., \& Marelli, M. (2019). Enter Sandman: Compound Processing and Semantic Transparency in a Compositional Perspective. Journal of Experimental Psychology: Learning, Memory, and Cognition, 45, 1872-1882.

Günther, F., \& Marelli, M. (2020). Trying to make it work: Compositional effects in the processing of compound "nonwords". Quarterly Journal of Experimental Psychology, 73, 1082-1091.

Günther, F., \& Marelli, M. (2021). CAOSS and transcendence: Modeling role-dependent constituent meanings in compounds. Morphology, Advance online publication. doi: https://doi.org/ 10.1007/s11525-021-09386-6

Günther, F., Petilli, M. A., Vergallito, A., \& Marelli, M. (2020). Images of the unseen: Extrapolating visual representations for abstract and concrete words in a data-driven computational model. Psychological Research, Advance online publication. doi: 10.1007/s00426-020-01429-7

Günther, F., Rinaldi, L., \& Marelli, M. (2019). Vector-space models of semantic representation from a cognitive perspective: A discussion of common misconceptions. Perspectives on Psychological Science, 14, 1006-1033.

Harris, Z. (1954). Distributional Structure. Word, 10, 146-162.

Hartung, J., \& Knapp, G. (2005). Multivariate multiple regression. In Encyclopedia of Statistics in Behavioral Science (Vol. 3, p. 1370-1373). Chichester, UK: Wiley.

Heylen, K., Wielfaert, T., Speelman, D., \& Geeraerts, D. (2015). Monitoring polysemy: Word space models as a tool for largescale lexical semantic analysis. Lingua, 157, 153-172.

Hollis, G., \& Westbury, C. (2016). The principals of meaning: Extracting semantic dimensions from co-occurrence models of semantics. Psychonomic Bulletin $\mathcal{E}$ Review, 23, 1744-1756.

Hollis, G., Westbury, C., \& Lefsrud, L. (2017). Extrapolating human judgments from skip-gram vector representations of word meaning. Quarterly Journal of Experimental Psychology, 70, 1603-1619.

Jackendoff, R. S. (2009). Compounding in the Parallel Architecture and Conceptual Semantics. In R. Lieber \& P. Štekauer (Eds.), 
The Oxford Handbook of Compounding (pp. 105-128). New York, NY: Oxford University Press.

Jamieson, R. K., Avery, J. E., Johns, B. T., \& Jones, M. N. (2018). An instance theory of semantic memory. Computational Brain $\mathcal{E}$ Behavior, 1, 119-136.

Ji, H., Gagné, C. L., \& Spalding, T. L. (2011). Benefits and costs of lexical decomposition and semantic integration during the processing of transparent and opaque English compounds. Journal of Memory and Language, 65, 406-430.

Johns, B. T., Jones, M., \& Mewhort, D. J. (2016). Experience as a free parameter in the cognitive modeling of language. In Proceedings of the 38th Annual Conference of the Cognitive Science Society (pp. 2291-2296). Austin, TX: Cognitive Science Society.

Jones, M. N., Willits, J., \& Dennis, S. (2015). Models of semantic memory. In J. Busemeyer, Z. Wang, J. Townsend, \& A. Eidels (Eds.), Oxford Handbook of Mathematical and Computational Psychology (pp. 232-254). New York, NY: Oxford University Press.

Juhasz, B. J., Lai, Y.-H., \& Woodcock, M. L. (2015). A database of 629 English compound words: ratings of familiarity, lexeme meaning dominance, semantic transparency, age of acquisition, imageability, and sensory experience. Behavior Research Methods, 47, 1004-1019.

Kay, P., \& Zimmer, K. (1976). On the semantics of compounds and genitives in English. In Sixth California Linguistics Association Proceedings (pp. 29-35).

Kim, S. Y., Yap, M. J., \& Goh, W. D. (2019). The role of semantic transparency in visual word recognition of compound words: A megastudy approach. Behavior Research Methods, 51, 27222731.

Kintsch, W. (2001). Predication. Cognitive Science, 25, 173-202.

Lake, B. M., \& Murphy, G. L. (2021). Word meaning in minds and machines. Psychological Review, Advance online publication. doi: https://doi.org/10.1037/rev0000297

Landauer, T. K., \& Dumais, S. T. (1997). A solution to Plato's problem: The Latent Semantic Analysis theory of acquisition, induction, and representation of knowledge. Psychological Review, 104, 211-240.

Lenci, A. (2008). Distributional semantics in linguistic and cognitive research. Italian Journal of Linguistics, 20(1), 1-31.

Lenci, A., Sahlgren, M., Jeuniaux, P., Gyllensten, A. C., \& Miliani, M. (2021). A comprehensive comparative evaluation and analysis of distributional semantic models.

Levi, J. N. (1978). The Syntax and Semantics of Complex Nominals. New York, NY: Academic Press.

Libben, G. (2014). The nature of compounds: A psychocentric perspective. Cognitive Neuropsychology, 31, 8-25.

Libben, G., Gibson, M., Yoon, Y. B., \& Sandra, D. (2003). Compound fracture: The role of semantic transparency and morphological headedness. Brain and Language, 84, 50-64.

Lynott, D., \& Ramscar, M. J. A. (2001). Can we model conceptual combination using distributional information? In Proceedings of the Twelfth Irish Conference on Artificial Intelligence and Cognitive Science (pp. 1-10). Maynooth, Ireland.

Mandera, P., Keuleers, E., \& Brysbaert, M. (2017). Explaining human performance in psycholinguistic tasks with models of semantic similarity based on prediction and counting: A review and empirical validation. Journal of Memory and Language, 92, 57-78.

Marelli, M., \& Baroni, M. (2015). Affixation in semantic space: Modeling morpheme meanings with compositional distributional semantics. Psychological Review, 122, 485-515.

Marelli, M., Dinu, G., Zamparelli, R., \& Baroni, M. (2015). Picking buttercups and eating butter cups: Spelling alternations, semantic relatedness, and their consequences for compound processing. Applied Psycholinguistics, 36, 1421-1439.

Marelli, M., Gagné, C. L., \& Spalding, T. L. (2017). Compounding as Abstract Operation in Semantic Space: A data-driven, largescale model for relational effects in the processing of novel compounds. Cognition, 166, 207-224.

Marelli, M., \& Luzzatti, C. (2012). Frequency effects in the processing of Italian nominal compounds: Modulation of headedness and semantic transparency. Journal of Memory and Language, 66, 644-664.

Martínez-Huertas, J. Á., Jorge-Botana, G., Luzón, J. M., \& Olmos, R. (2021). Redundancy, isomorphism, and propagative mechanisms between emotional and amodal representations of words: A computational study. Memory $\mathcal{E}$ Cognition, 49, 219-234.

McRae, K., Cree, G. S., Seidenberg, M. S., \& McNorgan, C. (2005). Semantic feature production norms for a large set of living and nonliving things. Behavior Research Methods, 37, 547-559.

Mikolov, T., Chen, K., Corrado, G., \& Dean, J. (2013). Efficient estimation of word representations in vector space. arXiv:1301.3781v3.

Mitchell, J., \& Lapata, M. (2010). Composition in Distributional Models of Semantics. Cognitive Science, 34, 1388-1439.

Murphy, G. L. (2002). Conceptual Combination. In G. L. Murphy (Ed.), The Big Book of Concepts (pp. 443-475). Cambridge, MA: MIT Press.

Pereira, F., Gershman, S., Ritter, S., \& Botvinick, M. (2016). A comparative evaluation of off-the-shelf distributed semantic representations for modelling behavioural data. Cognitive Neuropsychology, 33, 175-190.

Ran, B., \& Duimering, P. R. (2009). Conceptual Combination: Models, Theories, and Controversies. In S. P. Weingarten \& H. O. Penat (Eds.), Cognitive Psychology Research Developments (pp. 39-64). New York, NY: Nova Science.

Richie, R., Zou, W., \& Bhatia, S. (2019). Predicting high-level human judgment across diverse behavioral domains. Collabra: Psychology, 5(1), 50.

Rodd, J. M. (2020). Settling into semantic space: An ambiguityfocused account of word-meaning access. Perspectives on Psychological Science, 15, 411-427.

Rogers, T. T., \& Wolmetz, M. (2016). Conceptual knowledge representation: A cross-section of current research. Cognitive Neuropsychology, 33, 121-129.

Ryder, M. E. (1994). Ordered Chaos: The interpretation of English noun-noun compounds. Berkeley and Los Angeles, CA: University of California Press.

Schäfer, M., \& Bell, M. J. (2020). Constituent polysemy and interpretational diversity in attested English novel compounds. The Mental Lexicon, 15, 42-61.

Schmidtke, D., Gagné, C. L., Kuperman, V., \& Spalding, T. L. (2018). Language experience shapes relational knowledge of 
compound words. Psychonomic Bulletin E Review, 25, 14681487.

Schmidtke, D., Gagné, C. L., Kuperman, V., Spalding, T. L., \& Tucker, B. V. (2018). Conceptual relations compete during auditory and visual compound word recognition. Language, Cognition and Neuroscience, 33, 923-942.

Schmidtke, D., Kuperman, V., Gagné, C. L., \& Spalding, T. L. (2016). Competition between conceptual relations affects compound recognition: the role of entropy. Psychonomic Bulletin $\mathcal{E}$ Review, 23, 556-570.

Shwartz, V., \& Dagan, I. (2018). Paraphrase to explicate: Revealing implicit noun-compound relations. arXiv preprint; arXiv:1805.02442.

Snefjella, B., \& Blank, I. (2020). Semantic norm extrapolation is a missing data problem. PsyArXiv preprint. doi: 10.31234/osf.io/ y2gav

Snefjella, B., Lana, N., \& Kuperman, V. (2020). How emotion is learned: Semantic learning of novel words in emotional contexts. Journal of Memory and Language, 104171.

Sommerauer, P., \& Fokkens, A. (2018). Firearms and Tigers are Dangerous, Kitchen Knives and Zebras are Not: Testing whether Word Embeddings Can Tell. In Proceedings of the 2018 EMNLP Workshop BlackboxNLP: Analyzing and Interpreting Neural Networks for NLP (pp. 276-286). Brussels, Belgium: ACL.

Spalding, T. L., \& Gagné, C. L. (2014). Relational diversity affects ease of processing even for opaque english compounds. The Mental Lexicon, 9, 48-66.

Spalding, T. L., Gagné, C. L., Mullaly, A. C., \& Ji, H. (2010). Relation-based interpretation of noun-noun phrases: A new theoretical approach. Linguistische Berichte Sonderheft, 17, 283315.

Turney, P. D., \& Pantel, P. (2010). From frequency to meaning: Vector space models of semantics. Journal of Artificial Intelligence Research, 37, 141-188.

Utsumi, A. (2020). Exploring what is encoded in distributional word vectors: A neurobiologically motivated analysis. Cognitive Science, 44, e12844.

Vecchi, E. M., Marelli, M., Zamparelli, R., \& Baroni, M. (2017). Spicy adjectives and nominal donkeys: Capturing semantic deviance using compositionality in distributional spaces. Cognitive Science, 41, 102-136.

Verhoeven, B., \& Daelemans, W. (2013). Semantic classification of Dutch noun-noun compounds: A distributional semantics approach. Computational Linguistics in the Netherlands Journal, $3,2-18$.

Williams, E. (1981). On the notions "lexically related" and "head of a word". Linguistic Inquiry, 12, 245-274.

Zou, W., \& Bhatia, S. (2021). Judgment errors in naturalistic numerical estimation. Cognition, 211, 104647. 


\section{Supplementary Material A: Average performance of the mapping systems in Experiment 1 (detailed)}

\section{Experiment 1: Test compounds represented via CAOSS vectors}

Table 2

Average performance (measured as the correlation between the observed and predicted distributions of relational judgments) with .95-confidence intervals for training setups of the mapping system in Experiment 1, listed in order of performance. Left column: all negative values in the estimates set to zero, right column: standard regression model.

\begin{tabular}{l|cc|cc}
\hline mapping system trained on & $\begin{array}{c}\text { mean } \\
\text { (neg.-to-zero) }\end{array}$ & $\begin{array}{c}.95-C I \\
\text { (neg.-to-zero) }\end{array}$ & $\begin{array}{c}\text { mean } \\
\text { (standard) }\end{array}$ & $\begin{array}{c}.95-\mathrm{CI} \\
\text { (standard) }\end{array}$ \\
\hline whole-word & .428 & {$[.404, .453]$} & .411 & {$[.387, .435]$} \\
CAOSS & .399 & {$[.372, .427]$} & .354 & {$[.327, .382]$} \\
random whole-word & .276 & {$[.251, .302]$} & .265 & {$[.240, .291]$} \\
random CAOSS & .228 & {$[.202, .254]$} & .219 & {$[.193, .245]$} \\
completely random & .222 & {$[.194, .249]$} & .169 & {$[.141, .198]$} \\
additive & .211 & {$[.189, .232]$} & .210 & {$[.190, .230]$} \\
\hline
\end{tabular}

\section{Experiment 1: Test compounds represented via whole-word vectors}

Table 3

Average performance (measured as the correlation between the observed and predicted distributions of relational judgments) with .95-confidence intervals for training setups of the mapping system in Experiment 1, listed in order of performance. Left column: all negative values in the estimates set to zero, right column: standard regression model.

\begin{tabular}{l|cc|cc}
\hline mapping system trained on & $\begin{array}{c}\text { mean } \\
\text { (neg.-to-zero) }\end{array}$ & $\begin{array}{c}.95-\mathrm{CI} \\
\text { (neg.-to-zero) }\end{array}$ & $\begin{array}{c}\text { mean } \\
\text { (standard) }\end{array}$ & $\begin{array}{c}.95-\mathrm{CI} \\
\text { (standard) }\end{array}$ \\
\hline whole-word & .325 & {$[.298, .352]$} & .283 & {$[.256, .309]$} \\
CAOSS & .237 & {$[.210, .265]$} & .168 & {$[.142, .195]$} \\
random whole-word & .223 & {$[.196, .250]$} & .212 & {$[.185, .240]$} \\
additive & .200 & {$[.177, .224]$} & .175 & {$[.152, .198]$} \\
completely random & .168 & {$[.141, .194]$} & .091 & {$[.062, .119]$} \\
random CAOSS & .134 & {$[.107, .160]$} & .045 & {$[.017, .074]$} \\
\hline
\end{tabular}

\section{Experiment 1: Test compounds represented via additive model}

Table 4

Average performance (measured as the correlation between the observed and predicted distributions of relational judgments) with .95-confidence intervals for training setups of the mapping system in Experiment 1, listed in order of performance.Left column: all negative values in the estimates set to zero, right column: standard regression model.

\begin{tabular}{l|cc|cc}
\hline mapping system trained on & $\begin{array}{c}\text { mean } \\
\text { (neg.-to-zero) }\end{array}$ & $\begin{array}{c}.95-\mathrm{CI} \\
\text { (neg.-to-zero) }\end{array}$ & $\begin{array}{c}\text { mean } \\
\text { (standard) }\end{array}$ & $\begin{array}{c}.95-\mathrm{CI} \\
\text { (standard) }\end{array}$ \\
\hline additive & .344 & {$[.316, .372]$} & .300 & {$[.272, .328]$} \\
whole-word & .279 & {$[.253, .306]$} & .232 & {$[.207, .258]$} \\
CAOSS & .227 & {$[.200, .255]$} & .126 & {$[.099, .154]$} \\
random whole-word & .162 & {$[.135, .189]$} & .080 & {$[.050, .110]$} \\
completely random & .139 & {$[.112, .166]$} & .049 & {$[.021, .078]$} \\
random CAOSS & .112 & {$[.084, .139]$} & .002 & {$[-.027, .031]$} \\
\hline
\end{tabular}




\section{Supplementary Material B: Average performance of the mapping systems in Experiment 2 (detailed)}

\section{Experiment 2: Novel compounds represented via CAOSS model}

\section{Table 5}

Average performance (measured as the correlation between the observed and predicted distributions of relational judgments) with .95-confidence intervals for training setups of the mapping system in Experiment 2, listed in order of performance. Left column: all negative values in the estimates set to zero, right column: standard regression model.

\begin{tabular}{l|cc|cc}
\hline mapping system trained on & $\begin{array}{c}\text { mean } \\
\text { (neg.-to-zero) }\end{array}$ & $\begin{array}{c}.95-\mathrm{CI} \\
\text { (neg.-to-zero) }\end{array}$ & $\begin{array}{c}\text { mean } \\
\text { (standard) }\end{array}$ & $\begin{array}{c}.95-\mathrm{CI} \\
\text { (standard) }\end{array}$ \\
\hline whole-word & .296 & {$[.266, .327]$} & .278 & {$[.247, .308]$} \\
CAOSS & .290 & {$[.256, .324]$} & .253 & {$[.220, .287]$} \\
additive & .228 & {$[.200, .256]$} & .227 & {$[.200, .253]$} \\
random whole-word & .191 & {$[.161, .221]$} & .169 & {$[.139, .199]$} \\
completely random & .130 & {$[.100, .160]$} & .086 & {$[.055, .117]$} \\
random CAOSS & .123 & {$[.093, .154]$} & .074 & {$[.043, .106]$} \\
\hline
\end{tabular}

Table 6

Average performance (measured as the correlation between the observed and predicted distributions of relational judgments) with .95-confidence intervals for training setups of the mapping system in the subset analysis of Experiment 2, listed in order of performance. Top: Results for the subset of 204 compounds that share at least one constituent with the 575 existing compounds, bottom: Results for the subset of 204 compounds that share no constituent with these 575 existing compounds. Left column: all negative values in the estimates set to zero, right column: standard regression model.

\begin{tabular}{l|l|cc|cc}
\hline subset & mapping system trained on & $\begin{array}{c}\text { mean } \\
\text { (neg.-to-zero) }\end{array}$ & $\begin{array}{c}.95-\text { CI } \\
\text { (neg.-to-zero) }\end{array}$ & $\begin{array}{c}\text { mean } \\
\text { (standard) }\end{array}$ & $\begin{array}{c}.95-C I \\
\text { (standard) }\end{array}$ \\
\hline shared & CAOSS & .408 & {$[.364, .453]$} & .362 & {$[.316, .407]$} \\
const. & whole-word & .394 & {$[.351, .436]$} & .381 & {$[.340, .421]$} \\
& additive & .273 & {$[.234, .313]$} & .266 & {$[.229, .303]$} \\
& random whole-word & .261 & {$[.219, .303]$} & .241 & {$[.199, .283]$} \\
& completely random & .146 & {$[.101, .190]$} & .097 & {$[.052, .142]$} \\
& random CAOSS & .140 & {$[.097, .183]$} & .093 & {$[.047, .139]$} \\
\hline \hline no shared & whole-word & .199 & {$[.158, .239]$} & .175 & {$[.134, .216]$} \\
const. & additive & .182 & {$[.144, .221]$} & .187 & {$[.150, .224]$} \\
& CAOSS & .172 & {$[.125, .218]$} & .145 & {$[.101, .190]$} \\
& random whole-word & .121 & {$[.080, .161]$} & .097 & {$[.056, .138]$} \\
& completely random & .114 & {$[.073, .155]$} & .075 & {$[.032, .117]$} \\
& random CAOSS & .107 & {$[.064, .149]$} & .055 & {$[.012, .098]$} \\
\hline
\end{tabular}

Experiment 2: Novel compounds represented via the additive model

Table 7

Average performance (measured as the correlation between the observed and predicted distributions of relational judgments) with .95-confidence intervals for training setups of the mapping system in Experiment 2, listed in order of performance. Left column: all negative values in the estimates set to zero, right column: standard regression model.

\begin{tabular}{l|cc|cc}
\hline mapping system trained on & $\begin{array}{c}\text { mean } \\
\text { (neg.-to-zero) }\end{array}$ & $\begin{array}{c}.95-\mathrm{CI} \\
\text { (neg.-to-zero) }\end{array}$ & $\begin{array}{c}\text { mean } \\
\text { (standard) }\end{array}$ & $\begin{array}{c}.95-\mathrm{CI} \\
\text { (standard) }\end{array}$ \\
\hline additive & .257 & {$[.226, .289]$} & .217 & {$[.185, .248]$} \\
whole-word & .204 & {$[.173, .235]$} & .153 & {$[.121, .184]$} \\
CAOSS & .150 & {$[.118, .182]$} & .085 & {$[.053, .116]$} \\
random whole-word & .134 & {$[.104, .165]$} & .076 & {$[.045, .108]$} \\
completely random & .099 & {$[.067, .130]$} & .031 & {$[-.002, .065]$} \\
random CAOSS & .092 & {$[.063, .121]$} & .020 & {$[-.011, .051]$} \\
\hline
\end{tabular}


Table 8

Average performance (measured as the correlation between the observed and predicted distributions of relational judgments) with .95-confidence intervals for training setups of the mapping system in the subset analysis of Experiment 2, listed in order of performance. Top: Results for the subset of 204 compounds that share at least one constituent with the 575 existing compounds, bottom: Results for the subset of 204 compounds that share no constituent with these 575 existing compounds. Left column: all negative values in the estimates set to zero, right column: standard regression model.

\begin{tabular}{l|l|cc|cc}
\hline subset & mapping system trained on & $\begin{array}{c}\text { mean } \\
\text { (neg.-to-zero) }\end{array}$ & $\begin{array}{c}.95-\text {-CI } \\
\text { (neg.-to-zero) }\end{array}$ & $\begin{array}{c}\text { mean } \\
\text { (standard) }\end{array}$ & $\begin{array}{c}.95-\text {-CI } \\
\text { (standard) }\end{array}$ \\
\hline shared & additive & .365 & {$[.320, .410]$} & .337 & {$[.293, .381]$} \\
const. & whole-word & .275 & {$[.232, .318]$} & .230 & {$[.188, .272]$} \\
& CAOSS & .204 & {$[.157, .250]$} & .127 & {$[.082, .173]$} \\
& random whole-word & .154 & {$[.110, .198]$} & .090 & {$[.043, .137]$} \\
& random CAOSS & .101 & {$[.058, .143]$} & .021 & {$[-.023, .066]$} \\
& completely random & .066 & {$[.021, .110]$} & -.007 & {$[-.054, .039]$} \\
\hline \hline no shared & additive & .150 & {$[.110, .190]$} & .096 & {$[.056, .136]$} \\
const. & whole-word & .133 & {$[.090, .177]$} & .075 & {$[.029, .121]$} \\
& completely random & .132 & {$[.087, .176]$} & .070 & {$[.023, .117]$} \\
& random whole-word & .115 & {$[.072, .157]$} & .063 & {$[.020, .105]$} \\
& CAOSS & .096 & {$[.054, .138]$} & .042 & {$[.000, .085]$} \\
& random CAOSS & .084 & {$[.044, .124]$} & .019 & {$[-.024, .062]$} \\
\hline
\end{tabular}

